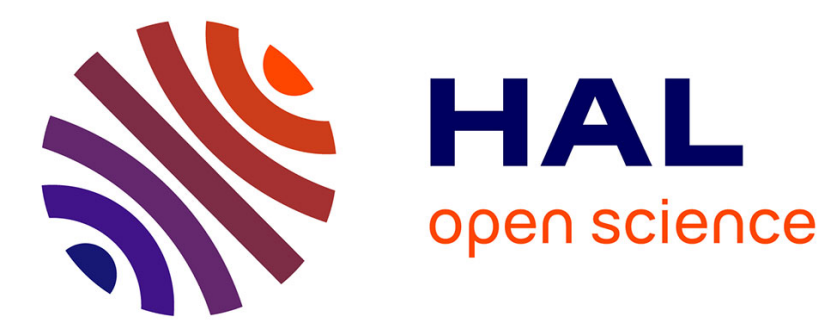

\title{
A human-like learning control for digital human models in a physics-based virtual environment
}

Giovanni de Magistris, Alain Micaelli, Paul Evrard, Jonathan Savin

\section{To cite this version:}

Giovanni de Magistris, Alain Micaelli, Paul Evrard, Jonathan Savin. A human-like learning control for digital human models in a physics-based virtual environment. Journal of Visualization and Computer Animation, 2015, 31, pp.423-440. 10.1007/s00371-014-0939-0 . hal-01171441

\section{HAL Id: hal-01171441 https://hal.science/hal-01171441}

Submitted on 3 Jul 2015

HAL is a multi-disciplinary open access archive for the deposit and dissemination of scientific research documents, whether they are published or not. The documents may come from teaching and research institutions in France or abroad, or from public or private research centers.
L'archive ouverte pluridisciplinaire HAL, est destinée au dépôt et à la diffusion de documents scientifiques de niveau recherche, publiés ou non, émanant des établissements d'enseignement et de recherche français ou étrangers, des laboratoires publics ou privés. 


\title{
A human-like learning control for digital human models in a physics-based virtual environment
}

\author{
Giovanni De Magistris · Alain Micaelli · Paul Evrard · Jonathan Savin
}

Received: / Accepted:

\begin{abstract}
This paper presents a new learning control framework for digital human models in a physicsbased virtual environment. The novelty of our controller is that it combines multi-objective control based on human properties (combined feedforward and feedback controller) with a learning technique based on human learning properties (human-being's ability to learn novel task dynamics through the minimization of instability, error and effort). This controller performs multiple tasks simultaneously (balance, non-sliding contacts, manipulation) in real time and adapts feedforward force as well as impedance to counter environmental disturbances. It is very useful to deal with unstable manipulations, such as tool-use tasks, and to compensate for perturbations. An interesting property of our controller is that it is implemented in cartesian space with joint stiffness, damping and torque learning in a multi-objective control framework. The relevance of the proposed control method to model human motor adaptation has been demonstrated by various simulations.
\end{abstract}

G. De Magistris

CEA, LIST, LSI, rue de Noetzlin, Gif-sur-Yvette, F-91190 France

Tel.: +33761308466

E-mail: giovanni_demagistris@hotmail.it

E-mail: giovanni.de-magistris@cea.fr

A. Micaelli

CEA, LIST, LSI, rue de Noetzlin, Gif-sur-Yvette, F-91190 France

P. Evrard

CEA, LIST, LSI, rue de Noetzlin, Gif-sur-Yvette, F-91190 France

J. Savin

Institut national de recherche et de sécurité (INRS), rue du Morvan, CS 60027, Vandœuvre-lès-Nancy, F-54519 France
Keywords Digital Human Model - Motion Control . Bio-Inspired Motor Control · Virtual Reality

\section{Introduction}

Digital human model (DHM) technique is rapidly emerging as an enabling technology and a unique line of research for the verification of human factors issues in industry, which is the general purpose of our work.

In order to evaluate the physical (biomechanical) aspects of working conditions, several software packages have been developed to facilitate ergonomic assessment, such as SAMMIE [55], JACK [4], Ergoman [59] and SANTOSHuman $[69,68]$. Simulations computed with these software packages usually rely on kinematic animation frameworks. Such frameworks use either prerecorded motions obtained by a tracking system and motion capture or interactive manual positioning of the DHM body through a mouse, menus and keyboard. In the first case, simulations are realistic but they require extensive instrumentation of a full scale mock-up of the future workstation or a similar existing one. They are extremely time consuming because of motion capture data processing [6]. Furthermore, their ability to predict complex human postures and movements for various sizes and dimensions in a timely and realistic manner is strictly dependent on the accuracy of the motion database. In the second case, simulations are clearly subjective (the designer, possibly with no specific skill in ergonomics, chooses arbitrarily a posture or trajectory). Again, they are time consuming (built up like a cartoon) and usually appear unnatural [13], even though these digital manikins possess semi-automatic controls provided by a set of behaviours, such as gazing, reaching, walking and grasping. These issues do 


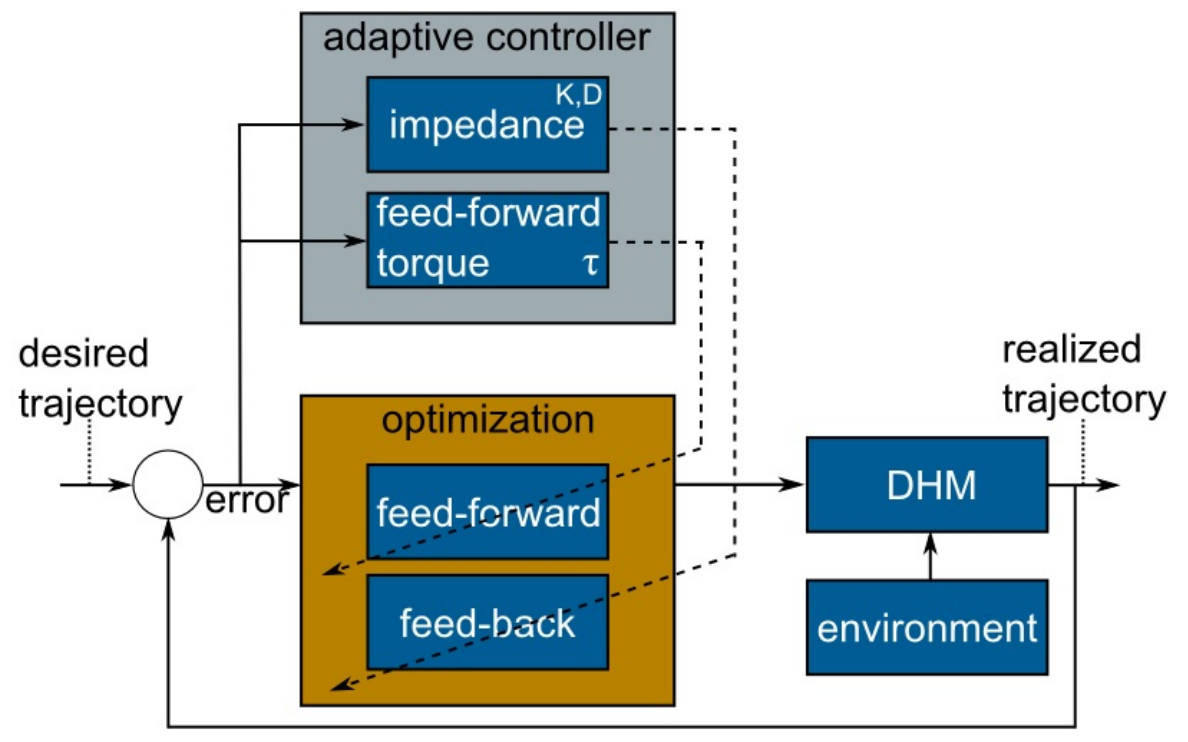

Fig. 1: Adaptive and Learning controller

not encourage designers to consider alternative scenarios, which would be beneficial for a comprehensive assessment of the future work situation. Moreover, such software packages are subject to numerous limitations: since they are restricted to static models and calculation, they neglect dynamic aspects. Neither do they consider contact forces between the DHM and objects (at best the designer has to arbitrarily set both contact force magnitude and direction manually). For these reasons, assessment of biomechanical risk factors based on simulations of industrial or experimental situations may lead to real stress underestimation of up to $40-50 \%$ [43].

A challenging aim therefore consists in developing a DHM capable of performing tasks as an artificial human-being through dynamically consistent motions, behaviours and internal characteristics (positions, velocities, accelerations and torques) based on a simple description of the future work task, in order to achieve realistic ergonomics assessments of various work task scenarii at an early stage of the design process.

\section{Human behaviours}

To achieve this goal, a multi-objective DHM controller based on human behaviours using simulated physics is presented in this article. In our simulation framework, the entire motion of the human model in the virtual environment is ruled by real-world Newtonian physical and mechanical simulation, along with automatic control of applied forces and torques. To develop this controller, we chose to take into account the following important behaviours of human motor control:
1. spring-like behaviour: Won and Hogan [71] noted that muscle elastic properties and reflexes produce a restoring force to an undisturbed trajectory when the hand is slightly perturbed, as a spring between the hand and the planned trajectory. The mechanical impedance (strength of these spring-like properties) increases with endpoint force [25] or muscle activation [39] and it is adapted to counter environmental disturbances [48]. This behaviour is implemented in the feedback part of our controller.

2. anticipatory capabilities: When a multibody system gets in touch with an object, it is important to make the limb more compliant to avoid "contact instability" [31]. An important conclusion, which consistently emerges from the theoretical analysis, is that mechanics needs a feedforward control.

A number of studies have shown that the nervous system uses internal representations to anticipate the consequences of dynamic interaction forces. In particular, Lackner and Dizio [40] demonstrated that the central nervous system (CNS) is able to predict the centripetal and Coriolis forces; Gribble and Ostry [26] demonstrated the compensation of interaction torques during multijoint limb movement. These studies suggest that the nervous system has sophisticated anticipatory capabilities. We therefore need to design accurate internal models of body dynamics and contacts.

Generally, a feedforward control model is based on the anticipatory computation of the forces that will be needed to carry out a desired motion plan, without sensory information. The CNS therefore needs 
an internal representation or an inverse model of the human model and environment.

This control technique is fast and does not have the instability risk, but has an obvious drawback: the sensitivity to unexpected disturbances. The feedforward control is not able to compensate for perturbations. If these disturbances can be measured, we can make on-the-fly correction of the movement. This method corresponds to the feedback control of our controller.

3. motion error minimization: Shadmehr and MussaIvaldi [61] demonstrated that, trial after trial, the CNS reduces motion error through the compensation of the environmental forces and the feedforward control adaptation. An illustrative example is Kawato's feedback error learning model [37], based on cooperation between two control mechanisms: a feedback loop, which operates in an initial training phase, and a feedforward model, which subsequently emerges. In this model, a feedback error is used as the learning signal for the feedforward model, which gradually compensates for any dynamic disturbances, and thereby learns an internal model of the body dynamics. This learning control model does not converge in unstable situations [52], while the controller described in this paper is more adapted to unstable interaction (see [72] and Sect. 9).

4. metabolic cost minimization: the CNS optimizes the arm impedance to achieve a desired margin of stability while minimizing metabolic cost [10].

Following these human motor control behaviours, we developed a new whole body control based on feedforward and feedback mechanisms (Fig. 1) inspired by the human ability to adapt force and impedance to deal with stable or unstable situations and to compensate for perturbations $[72,24]$.

\section{Overview on adaptive and learning control}

Adaptive and learning controls found in the literature can be distributed into four groups:

1. Classical Adaptive

- Gain Scheduling [2]

- Model Reference Adaptive Control (MRAC) [35]

- Self-tuning regulator [3]

- Self-Oscillating Adaptive Systems (SOAS) [33]

2. Periodic Adaptive/Learning

- Iterative Learning Control (ILC) [5]

- Repetitive Control (RC) [41]

- Run-to-Run control (R2R) [12]
3. Machine Learning

- Reinforcement Learning $[9,8]$

4. Non-symbolic learning tool

- Artificial neural network [32]

- Fuzzy logic [16]

- Genetic algorithms [62]

In our study, we wanted to develop an algorithm adapted to unstable interactions, which are inevitable in our context (namely, verification of human factors in industrial work task design). In particular, our case study dealt with the task of clipping small metal parts to a plastic instrument panel of a vehicle [19]. In this work-task, we observed subjects performing the same task repeatedly. When we tried to simulate this task with a DHM, one way to compensate for the repetitive part of the error is to use periodic adaptive/learning control. With this type of controller, a robot performs the same task for numerous iterations, reducing the periodic error at each following trial.

If a task has reproducible dynamics or fixed environment, impedance control is used to impose a desired dynamic behaviour to the interaction between the robot end-effector and the environment [30,14]. The common control impedance techniques requires a reproducible dynamics (the target impedance model is fixed). For this reason, it is not adapted when the environment changes (the interaction may become unstable).

One possible solution to perform unstable tasks is to increase impedance in order to deal with incorrect force arising from unknown dynamics. Yet, while higher impedance may increase stability in movement task, it may also lead to instability during interactions with a stiff environment.

Common periodic adaptive control learns only force from the feedback error. Thus, it is inefficient in unstable situations because the force will be different in each trial due to noise or external perturbations [70]. In addition, common ILC algorithms do not require a low mechanical impedance to obtain safety and energy minimization [28].

The algorithm developed below is more adapted to unstable interactions than common ILC algorithms, because it allows to change the force in each trial [11] and to obtain low impedance. Learning the optimal force and impedance appropriate for different tasks can help the robot achieve them with minimum error and least amount of energy (as humans do [23]). 


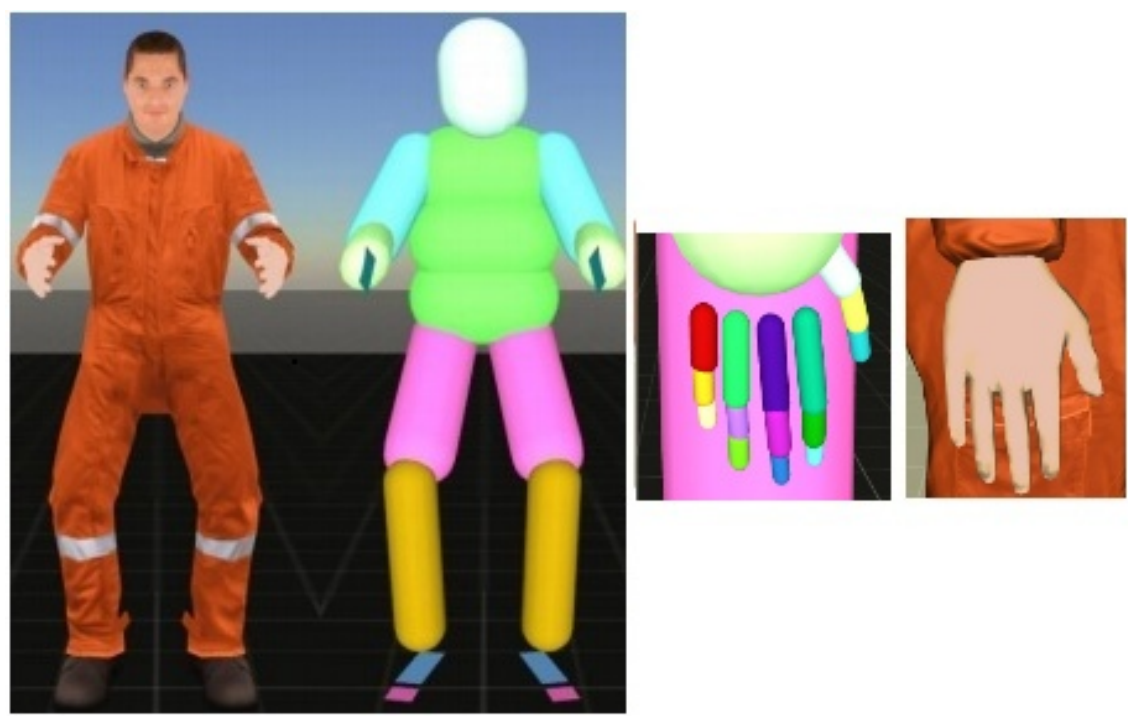

Fig. 2: DHM with skinning and collision geometry (left). Right hand model with skinning and collision geometry (right)

\section{Digital human model using simulated physics}

4.1 Model of human body and dynamics

In our study, the human body is kinematically modelled as a set of articulated rigid bodies (Fig. 2) organized into a redundant tree structure, which is characterized by its degrees of freedom (dof). Each articulation can be modelled into a number of revolute joints depending on the function of the corresponding human segment. Our DHM therefore comprises of 39 joint dof and 6 root dof, with 8 dof for each leg and 7 for each arm. The root is not controlled. For validation purposes, several DHMs have been dimensioned based on each subject's anthropometry [29].

The dynamics of the DHM are described as a second order system as:

$M \dot{T}+N T+G=L \tau+\sum_{j} J_{c_{j}}^{T} W_{c_{j}}+\sum_{k} J_{e n d_{k}}^{T} W_{e n d_{k}}^{i}$

$M$ is the generalized inertia matrix; $\dot{T}$ is the acceleration in generalized coordinates; $N T$ is the centrifugal and coriolis forces; $G$ is the gravity force in generalized coordinates; $L$ is the matrix to select the actuated degrees of freedom $\left(L=[0 I]^{T}\right.$ with 0 the zero matrix and $I$ the identity matrix); $\tau$ is the set of joint torques; $J$ is the jacobian matrix; $W$ is the wrench applied by digital human models on environments $\left(W=\left[\Gamma^{T} F^{T}\right]^{T}\right.$ with $\Gamma$ the moment in cartesian space and $F$ the force in cartesian space).

In the notation of this paper, frames are denoted by subscripts as follows:
- com: center of mass frame

- $c$ : non-sliding contacts at known fixed locations such as the contact points between the feet and the ground

- end: end-effector frame

- $q$ : joint space

- $\rho: \rho$-space

- $K, B, \tau$ : the learning rate of stiffness, damping or torque

Moreover the following superscripts are used:

- min: joint stiffness, damping and feedforward torque required to maintain posture stability and to reduce the systematic deviation caused by the interaction with the environment

- $d$ : "desired" values

- $l$ : the learned torque, stiffness or damping

- ini: the initial torque, stiffness or damping

- $i$ : wrench derived from unknown contacts with environment - interaction wrench

- ff: feedforward

- fb: feedback

- ob: object

\subsection{Contacts model}

Simulations were based on the XDE physics simulation module developed at the CEA-LIST (http://www.kalisteo.fr/lsi/en/aucune/a-proposde-xde). This module manages the whole physics simulation in real time, including accurate and robust 
constraint-based methods for contact and collision resolution $[45,46]$. Friction effects were modelled in compliance with Coulomb's friction law, which can be formulated as:

$\left\|f_{x y}\right\| \leq \mu\left\|f_{z}\right\|$

with $\left\|f_{x y}\right\|$ being the tangential contact force, $\mu$ the dry friction factor and $\left\|f_{z}\right\|$ the normal contact force.

\subsection{Hand model}

The hand model, illustrated in Fig. 2, has 20 dof. To control joint positions $\theta$, we use a simple ProportionalDerivative controller. The desired joint positions $\theta$ are a set of desired positions $\theta^{d}$ corresponding to different preset grasps.

\section{Adaptive controller based on human behaviours}

Corresponding to the above analysis of human motion control, we propose a human-like learning controller (Fig. 1) composed of feedforward and feedback controls, both of which are adapted during movements. This controller is inspired by the works of Yang et al [72] and Ganesh et al [24].

The proposed controller can deal with both stable and unstable conditions. The learned stiffness, damping and feedforward torque compensate for external perturbations. This behaviour is similar to human adaptation [65].

5.1 Cartesian controller with joint stiffness, damping and torque learning

We describe a cartesian controller that, given a target in cartesian space, learns joint space parameters. An interesting property of this controller is that although it is a cartesian controller, its impedance is learned and distributed according to the limbs' dynamics. As demonstrated in [44], to control limb stiffness and stability, the CNS must increase joint stiffness when an external force is applied to the hand. This result is obtained with our controller in Sect. 9.

The desired cartesian space impedance is:

$$
\begin{aligned}
& K_{\text {end }}=J_{\text {end, } \rho}^{\dagger T}\left(K_{\rho}-\frac{\partial J_{e n d, \rho}^{T}}{\partial \rho} W_{e n d}^{i}\right) J_{e n d, \rho}^{\dagger} \\
& B_{\text {end }}=J_{\text {end, } \rho}^{\dagger T} B_{\rho} J_{\text {end, } \rho}^{\dagger}
\end{aligned}
$$

$K$ is the stiffness matrix; $B$ is the damping matrix; $J^{\dagger}=M^{-1} J^{T}\left(J M^{-1} J^{T}\right)^{-1}$ is the dynamic pseudoinverse matrix with $\mathrm{J}$ a full rank matrix; $\rho=S q$. $S$ is a matrix to select a part of the actuated degrees of free$\operatorname{dom}\left(S=\left[\begin{array}{ll}I 0\end{array}\right]\right)$ to obtain a dyamic model independent of non-sliding contact forces at known fixed locations in Eq. 1 such as the contacts between the feet and the ground (see Appendix A).

\subsection{Overall cost function}

As explained in Sect. 2, the CNS minimizes the motion error cost $M_{E}(t)$ (Eq. 5) and the metabolic cost $M_{C}(t)$ [10] (to learn impedance and feedforward torque, a human does not spend extra effort (Eq. 6)). We therefore set our overall cost function $C(t)$ as:

$C(t)=M_{E}(t)+M_{C}(t)$

with:

$M_{E}(t)=\frac{1}{2} \epsilon^{T}(t)\left[J_{\text {end, } \rho}^{\dagger T} M_{\rho} J_{\text {end, },}^{\dagger}\right] \epsilon(t)$

and:

$M_{C}(t)=\frac{1}{2} \int_{t-D}^{t} \tilde{\Phi}^{T}(\sigma) Q^{-1} \tilde{\Phi}(\sigma) d \sigma$

$M_{\rho}$ is the inertia matrix (see Appendix A) and $Q=$ $\operatorname{diag}\left(I \otimes Q_{K}, I \otimes Q_{B}, Q_{\tau}\right)$.

$\epsilon$ is the tracking error commonly used in robotics [63] defined as:

$\epsilon=\delta\left(V^{d}, V^{r}\right)+b \delta\left(H^{d}, H^{r}\right) \in \operatorname{se}(3)$

with $H^{r} \in \mathrm{SE}(3), H^{d} \in \mathrm{SE}(3), V^{r} \in \operatorname{se}(3)$ and $V^{d} \in$ $\mathrm{se}(3)$, where $\mathrm{SE}(3)$ is the special Euclidian group and $\operatorname{se}(3)$ is the Lie algebra of $\mathrm{SE}(3)$.

$\delta\left(H^{d}, H^{r}\right)$ denotes the displacement (position and orientation) error between the desired and current state; $\delta\left(V^{d}, V^{r}\right)$ denotes the velocity (linear and angular velocity) error between the desired and current state.

$$
\begin{aligned}
\tilde{\Phi}(t)= & \Phi(t)-\Phi^{d}(t) \\
= & {\left[\operatorname{vec}\left(K_{\rho}^{l}(t)\right)^{T}, \operatorname{vec}\left(B_{\rho}^{l}(t)\right)^{T},\left(\tau_{\rho}^{l}(t)\right)^{T}\right]^{T} } \\
& -\left[\operatorname{vec}\left(K_{\rho}^{m i n}(t)\right)^{T}, \operatorname{vec}\left(B_{\rho}^{\min }(t)\right)^{T},\left(\tau_{\rho}^{\min }(t)\right)^{T}\right]^{T} \\
= & {\left[\operatorname{vec}(\tilde{K}(t))^{T}, \operatorname{vec}(\tilde{B}(t))^{T}, \tilde{\tau}(t)^{T}\right]^{T} }
\end{aligned}
$$

where $\operatorname{vec}(\cdot)$ is the column vectorization operator, $\tilde{K}=$ $K_{\rho}^{l}(t)-K_{\rho}^{\min }(t), \tilde{K}=B_{\rho}^{l}(t)-B_{\rho}^{\min }(t)$ and $\tilde{\tau}=$ $\tau_{\rho}^{l}(t)-\tau_{\rho}^{\min }(t) . K_{\rho}^{\text {min }}, B_{\rho}^{\text {min }}$ and $\tau_{\rho}^{\text {min }}$ are joint stiffness, damping and feedforward torque required to maintain posture stability and to reduce systematic deviation caused by the interaction with the environment (see Appendix B).

In Eq. 8, the function $\Phi(t)$ that adapts stiffness, damping and feedforward torque tends to the minimal value $\Phi^{d}(t)$ with a metabolic cost minimization [10]. 
To measure stability, we use the motion error cost $M_{E}$ in Eq. 5. If there exists $\delta>0$ such that

$\int_{t}^{t_{1}} \dot{M}_{E}(\sigma) d \sigma<\delta$

then human interaction with an environment is stable in $\left[t, t_{1}\right][36]$.

\subsection{DHM Torques}

Following the important behaviours of human motor control listed in Sect. 2, we propose a DHM controller composed of feedforward and feedback parts that are adapted during trials:

$\tau_{\rho}=S \tau^{f f}+S \tau^{f b}-\tau_{\rho}^{l}$

where $\tau^{f f}$ is the torque to compensate for DHM dynamics (feedforward part of our controller in Sect. 8.1); $\tau_{\rho}^{l}$ (Eq. 16) is the learned feedforward torque that depends on the feedback error.

$\tau^{f b}=-L^{T}\left(J_{\text {com }}^{T} F_{\text {com }}+J_{\text {end }}^{T} W_{\text {end }}^{d}+J_{c}^{T} \Delta f_{c}\right)$ is the torque to compensate trajectory errors (feedback part of our controller in Sect. 8.2). $\Delta f_{c}$ is the contact forces.

$W_{e n d}^{d}$ is the desired task wrench in Eq. 11 that adapts stiffness and damping in Eq. 8 .

The desired task wrench $W_{\text {end }}^{d}$ is computed by using an adaptive proportional-derivative (PD) feedback control law:

$W_{\text {end }}^{d}=K_{\text {end }}^{l} \delta\left(H^{d}, H^{r}\right)+B_{\text {end }}^{l} \delta\left(V^{d}, V^{r}\right)+B_{\text {end }}^{\text {ini }} \epsilon$

$=\left(K_{\text {end }}^{l}+b B_{\text {end }}^{\text {ini }}\right) \delta\left(H^{d}, H^{r}\right)+\left(B_{\text {end }}^{l}+B_{\text {end }}^{\text {ini }}\right) \delta\left(V^{d}, V^{r}\right)$

$K_{\text {end }}$ and $B_{\text {end }}$ denote the cartesian stiffness and damping matrix respectively.

As explained in Sect. 5.1, our controller learn joint space parameters using Eqs. 13 and 14. To pass from joint to cartesian stiffness and damping, we use the Eq. 3 . It is important to remember that joint-space and $\rho$-space are related by the relationship $\rho=S q$.

The $B_{\text {end }}^{\text {ini }}$ is chosen according to:

$B_{\text {end }}^{i n i}=J_{\text {end, } \rho}^{\dagger T} B_{\rho}^{i n i} J_{\text {end, } \rho}^{\dagger}$

with $B_{\rho}^{i n i}$ being a symmetric positive definite matrix with minimal eigenvalue $\lambda_{\min }\left(B_{\rho}^{i n i}\right) \geq \lambda_{B}>0$. This minimal feedback matrix insures stable and compliant motion control. It corresponds to the mechanical properties of the passive muscles of the human relaxed arm [54].

\subsection{Learning laws}

In order to vary the mechanical control of a limb over time, the cerebellum plays an important role in the human motor learning process, forming and storing associated muscle activation patterns. According to Smith [64], stiffness varies throughout the movement. Based on human properties detailed in Sect. 2, stiffness $K_{\rho}^{l}(t)$ and damping $B_{\rho}^{l}(t)$ are adapted as follows:

$K_{\rho}^{l}(t, k+1)=K_{\rho}^{l}(t, k)$

$+Q_{K}\left\{J_{\text {end, } \rho}^{\dagger}\left[\epsilon(t, k) \delta\left(H^{d}, H^{r}\right)^{T}\right] J_{e n d, \rho}^{\dagger T}-\gamma(t) K_{\rho}^{l}(t, k)\right\}$

$B_{\rho}^{l}(t, k+1)=B_{\rho}^{l}(t, k)$

$+Q_{B}\left\{J_{\text {end, } \rho}^{\dagger}\left[\epsilon(t, k) \delta\left(V^{d}, V^{r}\right)^{T}\right] J_{\text {end, } \rho}^{\dagger T}-\gamma(t) B_{\rho}^{l}(t, k)\right\}$

with $K_{\rho}^{l}(t, k=0)=0_{\left[n_{\rho}, n_{\rho}\right]}$ and $B_{\rho}^{l}(t, k=0)=0_{\left[n_{\rho}, n_{\rho}\right]}$, $t \in[0, D), Q_{K}$ and $Q_{B}$ are symmetric positive definite constant gain matrices.

The forgetting factor of learning $\gamma$ is defined by:

$\gamma(t)=\frac{p}{1+u\|\epsilon(t)\|^{2}}$

with positive $p$ and $u$ values. To obtain convergence, we need $\gamma(t)>0$ (see Appendix B). The learning response speed can be tuned through the choice of $p$ and $u$. If $\gamma(t)$ is large, torque and impedance learning will be slow; if $\gamma(t)$ is small, we will obtain slow torque and impedance unlearning.

Unlike the constant value of $\gamma$ in [24], the time varying definition of $\gamma$ in Eq. 15 has the following advantage: when $\epsilon(t)$ is large, $\gamma(t)$ is small and vice versa. For this reason, we have a controller that quickly increases torque and impedance during bad tracking performance and quickly decreases torque and impedance during good tracking performance.

The learned feedforward torque is adapted through:

$\tau_{\rho}^{l}(t, k+1)=\tau_{\rho}^{l}(t, k)+Q_{\tau}\left[J_{\text {end, }, \rho}^{\dagger} \epsilon(t, k)-\gamma(t, k) \tau_{\rho}^{l}(t, k)\right]$

with $\tau_{\rho}^{l}(t, k=0)=0_{\left[n_{\rho}, 1\right]}, t \in[0, D], Q_{\tau}$ a symmetric positive definite constant matrix.

The diagonal learning rate matrices $Q_{K}, Q_{B}$ and $Q_{\tau}$ are empirically chosen. In particular we choose $Q_{K}>Q_{\tau}$ because human stiffness increases faster than feedforward torque [10] and $Q_{B}=Q_{K} / b$ according to Eq. 11.

\section{Trajectory planner based on human psychophysical principles}

A movement can be characterized, independently of the end-effector, by:

- the initial and final points of the trajectory (position and orientation) 
- obstacle positions (via-points of the trajectory)

- duration

Experimental study of human movements has shown that voluntary movements obey the following three major psychophysical principles:

- Hick-Hyman's law: the average reaction time $T_{R_{\text {ave }}}$ required to choose among $n$ probable choices depends on their logarithm [34]:

$$
T_{R_{\text {ave }}}=d \log _{2}(n+1)
$$

- Fitts' law: the movement time depends on the logarithm of the relative accuracy (the ratio between movement amplitude and target dimension) [21]:

$$
D=g+z \log _{2}(2 \Upsilon P)
$$

where $D$ is the duration time, $\Upsilon$ is the amplitude, $P$ is the accuracy, and $g$ and $z$ are empirically determined constants.

- Kinematics invariance: hand movements have a bell-shaped speed profile in straight reaching movements [50]. The speed profile is independent of the movement direction and amplitude. For more complex trajectories (i.e. handwriting) the same principle predicts a correlation between speed and curvature [51] described as a $2 / 3$ power law:

$$
\dot{s}(t)=Z_{s} R^{1-\frac{2}{3}}
$$

where $\dot{s}(t)$ is the tangential velocity, $R$ is the radius of curvature and $Z_{s}$ is a proportionality constant, also termed "velocity gain factor".

For this reason, more complex trajectories can be divided into overlapping basic trajectory similar to reaching movements. Such spatio-temporal invariant features of normal movements can be explained by a variety of criteria of maximum smoothness, such as the minimum jerk criterion [22] or the minimum torque-change criterion [67].

We implemented a modified minimum jerk criterion with via-points to calculate trajectories and avoid obstacles.

The original minimum-jerk model in [22] may fail to predict the hand path and can only be applied to average data because it predicts a single optimum movement for given via-points. Unlike the original minimum jerk model, the $2 / 3$ power law can be applied to all movements. The main problem with this method is the formula, which predicts speed from paths. In this study, we therefore chose Todorov's model [66], which combines the original minimum-jerk model and the $2 / 3$ power law model and uses a path observed in a specific trial to predict the speed profile. Todorov's model substitutes a smoothness constraint for the $2 / 3$ power law (see Appendix C.2). This model is validated and compared to the $2 / 3$ power law in [66] for four tasks with a specified path.

For a given hand path in space, Todorov's model [66] assumes that the speed profile is the one that minimizes the third derivative of position (also named "jerk"):

$$
\operatorname{Jerk}=\int_{0}^{D}\left\|\frac{d^{3}}{d t^{3}} \mathbf{r}[s(t)]\right\|^{2}
$$

with $r(s)=[x(s), y(s), z(s)]$ a $3 \mathrm{D}$ hand path and $s$ is the curvilinear coordinate. According to this approach, minimization is performed only over the speed profiles because the path is specified. Formal definition of the inside term of the integral in Eq. 20 is in Appendix C.1.

In the original minimum jerk model [22], the minimum jerk trajectory is a 5th-order polynomial in $t$. Using the end-point constraints, we can compute the coefficients of this polynomial. The trajectory and speed are found by a given set of via-points and thus, the hand is constrained to pass through the via-points at definite times. To calculate the minimum jerk trajectory, it is necessary to give passage times $T_{P}$, positions $x$, velocities $v$ and accelerations $a$. In the Todorov's model, the passage times $T_{P}$ are not defined a priori, but are determined by the algorithm explained below.

To find the optimal jerk for any given passage times $T_{P}$ and intermediate points $x$, Todorov's model minimizes the jerk with respect to $v$ and $a$ by setting the gradient to zero and solving the resulting system of linear equations. To find the intermediate times $T_{P}$, the method uses a nonlinear simplex method to minimize the optimal jerk over all possible passage times.

In the same way as for translations, the speed profile of a rotation is the one that minimizes the third derivative of orientation (or "jerk"), with a $3 D$ rotation path $r(s)=[\alpha(s), \beta(s), \gamma(s)]$.

In brief, to calculate the minimum jerk trajectory for the rotations and the translations, we need to provide the positions $X$, the initial and final velocities $V$ and the initial and final accelerations $A$.

An illustrative example of a minimum jerk trajectory simulation is given in [19] and a comparison between real human data and simulations is given in [20].

\section{Duration time based on human laws}

Duration times are a-priori chosen following the 3D Fitt's law proposed in [27] for a pointing task. The reach and position states are similar to a pointing task at trivariate targets, and therefore we use the equation in 


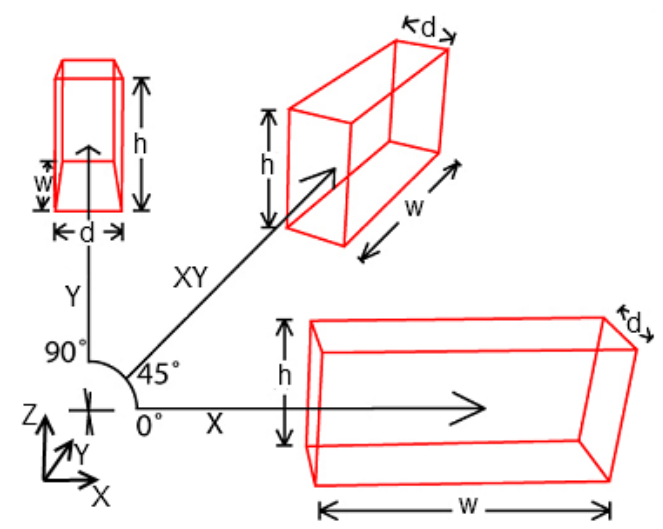

Fig. 3: w, h and d measurements for the 3D Fitt's law

[27] to calculate movement time $D$ :

$D \approx 56+5208 \log _{2}\left(\sqrt{f_{w}(\theta)\left(\frac{\gamma}{w}\right)^{2}+\frac{1}{9.2}\left(\frac{\gamma}{h}\right)^{2}+f_{d}(\theta)\left(\frac{r}{d}\right)^{2}}+1\right)$

with $f_{w}\left(0^{\circ}\right)=0.211, f_{w}\left(45^{\circ}\right)=0.242, f_{w}\left(90^{\circ}\right)=$ $0.717, f_{d}\left(0^{\circ}\right)=0.194, f_{d}\left(45^{\circ}\right)=0.147$ and $f_{d}\left(90^{\circ}\right)=$ 0.312 . $\Upsilon$ is the distance (or amplitude), $\theta$ is the movement angle (the human user's axis of movement), $w$ is the width measured along movement axis, $h$ is the height measured along Z-axis, and $d$ is perpendicular to both (see Fig. 3).

\section{Feedforward and feedback control}

The optimization framework (see Fig. 4) is based on a combined anticipatory feedforward and feedback controls system based on underlying notions of the acceleration - based control method $[1,15]$ and a JacobianTranspose (JT) control method $[57,42,18]$.

These controllers are formulated as two successive Quadratic Programming (QP) controllers (Fig. 4), each of them dealing with a great number of dof and solving simultaneously all constraint equations.

The controller is introduced to compute joint torques that achieve different objectives and satisfies different constraints. In our multi-objective control, a task means that a certain frame on the DHM body should be transferred from an initial state to a desired state.

\subsection{Feedforward}

During the feedforward phase, the objectives are:

1. Objective based on acceleration control. This feedforward action compensates for the low frequency, rigid body behaviour of the DHM dynamics. The goal is to minimize the difference between actual acceleration $A$ and desired acceleration $A^{d}$ found by the minimum jerk trajectory planner.

$A$ is expressed in terms of the unknowns of the system $\dot{T}$ as:

$$
\left\{\begin{array}{l}
V=J T \\
A=J \dot{T}+\dot{J} T
\end{array}\right.
$$

with $J$ being the Jacobian matrix expressed in its own frame.

2. Regularization for $Q P$ problem: To regularize the QP problem, we set the desired torque $\tau^{d}$, the desired contact force $f_{c}^{d}$ and the desired acceleration $\dot{T}^{d}$ to zero.

During the feedforward phase, the constraints are:

1. Dynamic equation. As explained in Sect. 2, the CNS is able to predict dynamics. We therefore set the DHM dynamics in Eq. 1 as a feedforward constraint.

2. Contact point accelerations. To help maintain contacts, contact acceleration must be null.

$A_{c}=J_{c} \dot{T}+\dot{J}_{c} T=0$

3. Non-sliding contacts. The non-sliding contacts are expressed as a set of inequality constraints. Contact constraints are imposed at contact points between the feet and the ground. The contact force $f_{c}$ should remain within the friction cone. The linearized Coulomb friction model [1] is applied, in which the friction cone of each contact is approximated by a four-faced polyhedral convex cone. The contact constraints are formularized as:

$E_{c_{i}} f_{c_{i}}+d_{c_{i}}<0$

where $E_{c_{i}}$ is the approximated friction cone, and $d_{c_{i}}$ is a customer defined margin vector so that the projection of $f_{c_{i}}$ on the normal vector of each facet of the friction cone should be kept larger than $d_{c_{i}}$. 


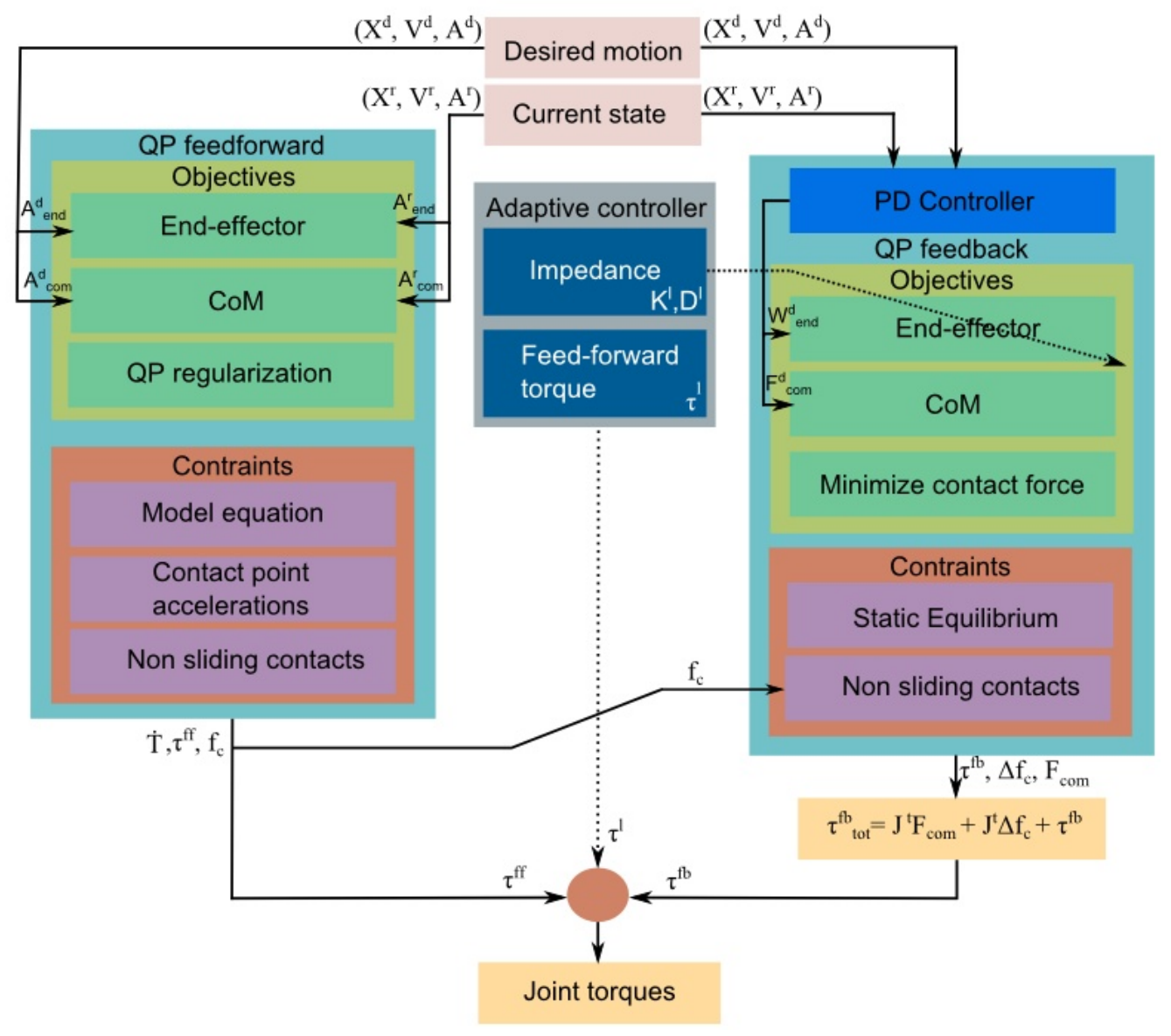

Fig. 4: Block diagram of the cartesian control framework

We summarize the feedforward phase as:

$$
\hat{O}=\underset{\tau^{f f}, \dot{T}, f_{c}}{\arg \min \frac{1}{2}}\left\|\left[\begin{array}{c}
\tau^{f f} \\
\dot{T} \\
f_{c}
\end{array}\right]-\left[\begin{array}{c}
\tau^{f f} d \\
\dot{T}^{d} \\
f_{c}^{d}
\end{array}\right]\right\|_{Q}^{2}
$$

subject to:

$$
\left\{\begin{array}{l}
M \dot{T}+N T+G=L \tau+J_{c}^{T} f_{c} \\
E_{c} f_{c}+d_{c} \geq 0 \\
J_{c} \dot{T}+\dot{J}_{c} T=0
\end{array}\right.
$$

The optimization objective is the same for each task, which is to minimize the error between the variable and its desired value. The objectives are combined in the diagonal weight matrix $\mathrm{Q}$. These values are chosen according to the priorities of different objectives.

With this optimization, we obtain $\tau^{f f}, f_{c}, \dot{T}$.

\subsection{Feedback}

In the feedback part, for each task, we imagine that a virtual wrench is applied at a certain frame on the
DHM body to guide its motion towards a given target. These virtual wrenches are computed by solving an optimization problem.

To obtain this, in the feedback phase the objectives are:

1. COM position. The dynamic controller maintains the DHM balance by imposing that the horizontal plane projection of the COM lie within a convex support region [7]. For this COM-tracking objective, we consider only the force component and $F_{\text {com }}^{d}$ is obtained by using a PD control in $\Re^{3}$ to measure the error between the actual and desired COM positions.

$F_{c o m}^{d}=K_{c o m}\left(x_{c o m}^{d}-x_{c o m}^{r}\right)+B_{c o m}\left(v_{c o m}^{d}-v_{c o m}^{r}\right)$

where $K_{\text {com }}$ and $B_{\text {com }}$ are the proportional and derivative gain matrix respectively.

2. End-effector. The end-effector task is used for performing some specific motions. In this paper the objective is to realize point to point movement with the human-based learning controller in Eq. 11. 
3. Minimize the difference between actual contact force and feedforward contact force. $\Delta f_{c}^{d}=0_{\left(3 n_{f_{c}}, 1\right)}$ with $Q_{\Delta f_{c}}=w_{\Delta f_{c}} I_{3 n_{f_{c}}}$

In the feedback phase the constraints are:

1. Static equilibrium. The wrenches are constrained by the static equilibrium of the DHM:

$$
L \tau^{f b}=-J_{\text {com }}^{T} F_{c o m}-J_{e n d}^{T} W_{e n d}^{d}-\sum_{i} J_{c_{i}}{ }^{T} \Delta f_{c_{i}}
$$

\section{Non-sliding contacts}

$$
E_{c}\left(f_{c}+\Delta f_{c}\right)+d_{c} \geq 0
$$

We summarize the feedback phase as:

$\hat{O}=\underset{F_{c o m}, \tau^{f b}, \Delta f_{c}}{\arg \min \frac{1}{2}}\left\|\left[\begin{array}{c}F_{\text {com }} \\ W_{\text {end }} \\ \Delta f_{c}\end{array}\right]-\left[\begin{array}{c}F_{c o m}^{d} \\ W_{e n d}^{d} \\ \Delta f_{c}^{d}\end{array}\right]\right\|_{Q}^{2}$

subject to:

$$
\left\{\begin{array}{l}
L \tau^{f b}=-J_{c o m}^{T} F_{c o m}-J_{e n d}^{T} W_{e n d}^{d}-J_{c}^{T} \Delta f_{c} \\
E_{c}\left(f_{c}+\Delta f_{c}\right)+d_{c} \geq 0
\end{array}\right.
$$

The optimization objective is the same for each task, which is to minimize the error between the variable and its desired value. The objectives are combined in the diagonal weight matrix $Q$. These values are chosen according to the priorities of different objectives.

With this optimization, we obtain $F_{c o m}, W_{\text {end }}, \Delta f_{c}$.

The feedback joint torque is equal to:

$\tau^{f b}=-L^{T}\left(J_{\text {com }}^{T} F_{c o m}+J_{\text {end }}^{T} W_{\text {end }}^{d}+J_{c}^{T} \Delta f_{c}\right)$

\section{Results}

Our simulation framework requires a PC running a Python 2.7 environment with XDE modules.

With a simulation step of $0.01 \mathrm{~s}$, the joint torques are calculated in quasi-real-time (computation duration is 1.5 times the simulation duration) on a $\mathrm{PC}$ equipped with an Intel Xeon E5630 (12M Cache, 2.53 GHz Processor, $24 \mathrm{~Gb}$ of RAM).

Several simulations have been made using our new joint stiffness, damping and torque-learning cartesian controller. A first case-study dealt with a fictional hand task, a second case-study dealt with an experimental assembly task. All simulations consisted of controlling a 45 dof DHM, with 6 dof for the root position and orientation, using actuators/muscle producing joint torques $\tau$ in a 6 -dimensional Cartesian task space characterized by an interaction external wrench $W_{e n d}^{i}$ while tracking a minimum-jerk task reference trajectory detailed in Sect.
6. The wrench is derived from the contacts or given by an imposed wrench field.

There are four contact points on each foot.

During the experimental task, we observed that torso orientation varied very little. We therefore add an objective to maintain the desired torso orientation equal to its initial orientation.

The optimization weights for the different objectives are: $10^{4}$ for the COM, $5 \cdot 10^{3}$ for the right hand task, $10^{1}$ for the posture, $10^{2}$ for the head, $10^{2}$ for the torso, $10^{0}$ for the contact task and $10^{2}$ for the gravity compensation. These weights are empirically chosen based on the estimated importance and priorities of the different objectives.

The learning rate matrices $Q_{K}, Q_{B}$ and $Q_{\tau}$ in [72] have been changed for different applications and they are empirically chosen based on the importance and priorities of the different objectives.

We choose $Q k>Q_{\tau}$ because human stiffness increase faster than feedforward torque [10]. The controller parameters are selected as $Q_{K}=\operatorname{diag}[8 .]_{\left(n_{\rho}, n_{\rho}\right)}$, $Q_{B}=\operatorname{diag}[0.8]_{\left(n_{\rho}, n_{\rho}\right)}, Q_{\tau}=\operatorname{diag}[1 .]_{\left(n_{\rho}, n_{\rho}\right)}, a=0.2$, $u=5, b=10$ for all simulations.

In [20], we used this controller to simulate an experimental insert clipping activity in quasi-real-time and applied the simulated postures, time and exertions to an OCRA index-based ergonomic assessment [53]. Given only scant information on the scenario (typically initial and final operator-positions and clipping force), the simulated ergonomic evaluations were in the same risk area as those based on experimental human data. In addition, DHM trajectories are similar to real trajectories.

\subsection{Free hand movement}

The first case studied is a point-to-point movement: the right hand goes from the initial right hand position to the insert position. At the start of the simulation, the insert is placed on the table in Fig. 8a, and the DHM body is upright and its arms are along the body. This reproduces the grasping action of the experimental task in [19].

The movement duration $D=1.3 \mathrm{~s}$ is chosen according to the 3D Fitt's law proposed in Sect. 7 for a pointing task.

A constant interaction external wrench $W_{\text {end }}^{\text {ext }}=$ $[0 N \cdot m, 0 N \cdot m, 0 N \cdot m, 3 N, 3 N, 3 N]^{T}$ is applied to the right hand during the motion. Adaptation is simulated for 225 iterations. At the end of each iteration the joint position is reset to the start point and the joint velocity and acceleration are reset to zero. 
In the first phase (iterations 1-75) interaction wrench is absent. In the second phase (iterations 76149) a constant perturbation wrench $W_{\text {end }}^{i}=W_{\text {end }}^{\text {ext }}$ is applied to the right hand. In the third phase (iterations 150-225) interaction wrench is absent.

As demonstrated in Fig. 5, DHM increases its joint stiffness in order to maintain limb stability in the presence of applied external forces at the hand $[10,44]$.

We note that the error decreases (see Fig. 6) and therefore, initial divergent trajectories become convergent and successful after learning.

We observe similar pattern of stiffness and feedforward torque (Fig. 7) of the experiments in [10]. This is derived from stiffness and damping adaptation to compensate for unstable interaction, without a large modification of the feedforward torque.

We note that the limb stiffness converges to small values when no forces are applied at the hand. The lower-magnitude joint stiffness is typical of a human subject acting with a zero force field [44].

\subsection{Simulation of a insertion with a virtual object}

In the second case-study, we simulate the insertion task in [19]. The interaction external wrench is derived from the contacts between the insert and the virtual object represented in Fig. 8a.

The digital mock-up (DMU) scenario is represented in Fig. 8a. This reproduces the experimental environment in [19] by ensuring geometric similarity. The inputs used to build the DMU scenario are the workplace spatial organization ( $\mathrm{x}, \mathrm{y}$ and $\mathrm{z}$ dimensions), inserts and tool descriptions ( $\mathrm{x}, \mathrm{y}, \mathrm{z}$ positions and weight) and the DHM position.

In Figs. 9 and 8b we show the results of the simulation when the right hand goes from the virtual object center $x^{o b}$ to the $x=x^{o b}+[0 \mathrm{~m}, 0.03 \mathrm{~m},-0.02 \mathrm{~m}]$ position (the reference frame is represented in Fig. 8a).

Adaptation is simulated for 50 iterations.

We note in Fig. 8b that the asymptotic force slightly decreases. We have demonstrated this human behavior by human subject experiments [17].

\section{Conclusion and future works}

In this paper, we have described a multi-objective control of digital human models based on human-being's ability to learn novel task dynamics through the minimization of instability, error and effort. Our controller has been validated with a 45 dof DHM. For this paper, we applied our algorithm to a rather simple case study, of limited impact relatively to the complexity of actual work gestures. In order to confirm the encouraging results and to give the desired genericity to our controller and DHM, we plan to do additional theoretical and practical works.

One improvement will be to enrich prehension simulation. For our case study, we explicitly specified the type of grasp (palmar, pinch, full-handed) and orientation of the object in the operator's hand, according to the final orientation (the object is attached to the hand). In the near future, we plan to introduce prehension functions in our kinematic model. In order not to make our kinematic model heavier (20 segments and 28 additional dof per hand [49]), we propose to replace the wrist and fingers by a dedicated end-effector whose characteristics (number of joints, types, rotational and translational range) would mimic the dof observed for each type of grasp [47]. For example, this effector would have more dof in pinch mode than in full-handed grasp mode.

Another improvement will involve the parametrization of the controller. Actually, our controller needs several parameters to be set, for instance tasks weights. In our case study, we set those parameters empirically. To improve the genericity of our algorithm, the tasks weights could be automatically calculated without requiring any manual tuning [58].

\section{References}

1. Abe, Y., Silva, M.D., Popović, J.: Multiobjective control with frictional contacts. In: Proc. ACM SIGGRAPH/EG Symposium on Computer Animation, pp. 249-258. AirelaVille, Switzerland (2007)

2. Andreiev, N.: A process controller that adapts to signal and process conditions. Control Engineering 38 (1977)

3. Astrom, K., Borrison, U., Wittenmark, B.: Theory and application of self-tuning regulators. Automatica 13, 457476 (1977)

4. Badler, N.: Virtual humans for animation, ergonomics, and simulation. In: Proceedings of the IEEE workshop on nonrigid and articulates motion, pp. 28-36 (1997)

5. Bien, Z., Xu, J.: Iterative learning control: analysis, design, integration and applications. In: Kluwer Academic Publishers Norwell. MA, USA (1998)

6. Bradwell, B., Li, B.: A tutorial on motion capture driven character animation. In: Eight IASTED International Conference Visualization, Imaging, and Image Processing. Palma de Mallorca (2008)

7. Bretl, T., Lall, S.: Testing static equilibrium for legged robots. IEEE Transactions on Robotics 24, 794-807 (2008)

8. Buchli, J., Stulp, F., Theodorou, E., Schaal, S.: Learning variable impedance control. The International Journal of Robotics 30, 820-833 (2011)

9. Buchli, J., Theodorou, E., Schaal, S.: Reinforcement learning of full-body humanoid motor skills. In: 10th IEEERAS International Conference on Humanoid Robots (Humanoids), pp. 405-410 (2010) 


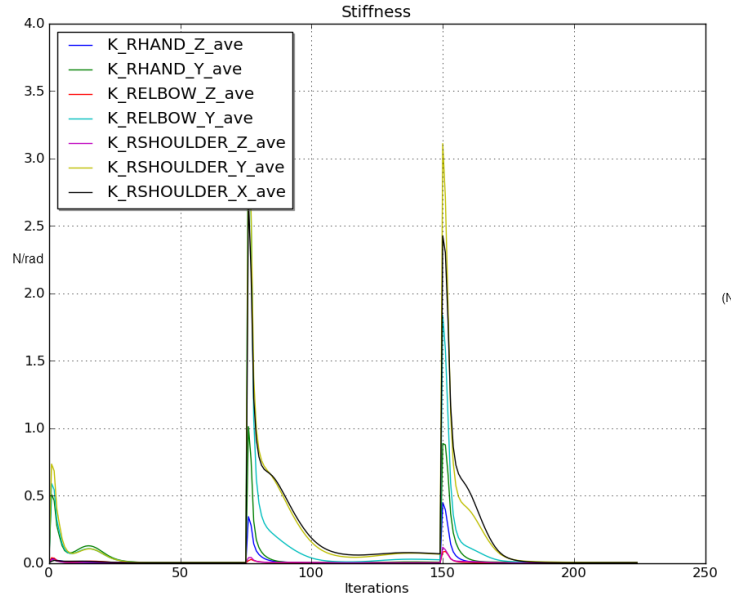

(a) Joint stiffness

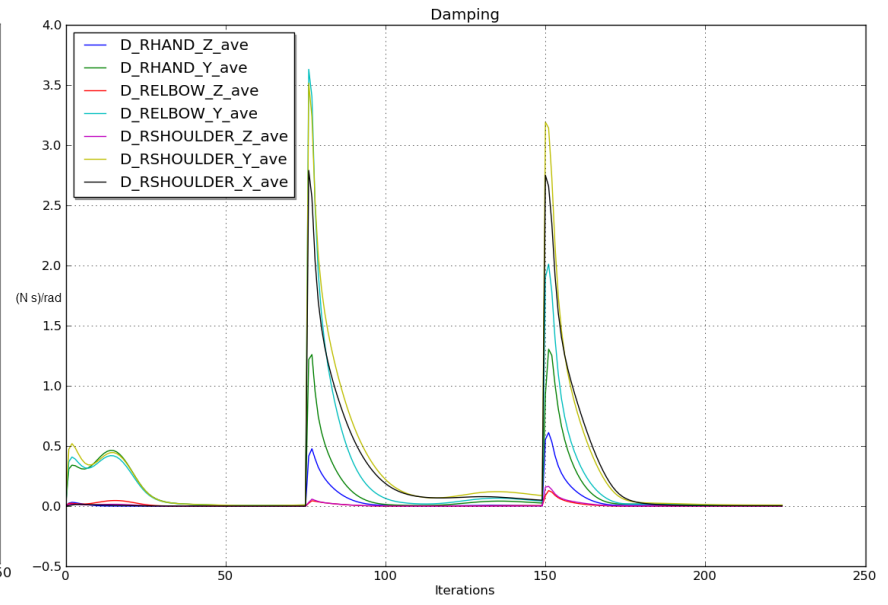

(b) Joint damping

Fig. 5: Learned joint stiffness and damping (mean over one period)

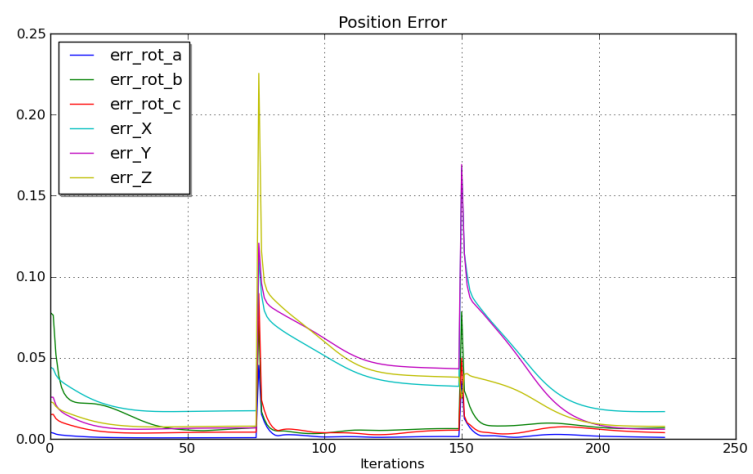

(a)

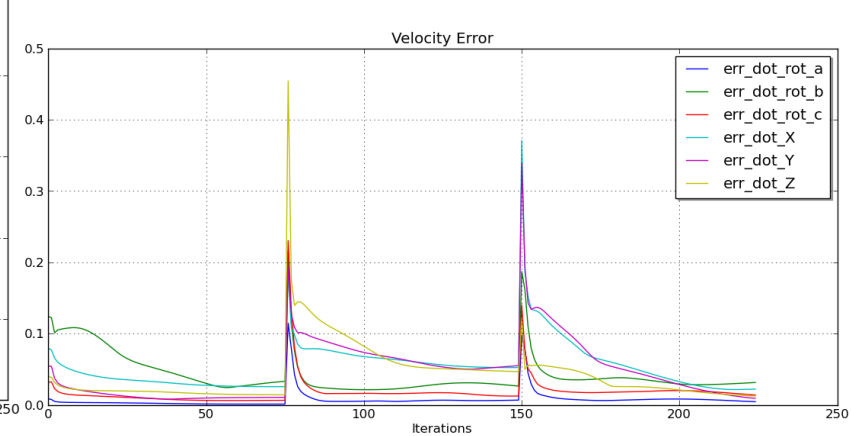

(b)

Fig. 6: Cartesian position [m] and orientation [rad] error (mean over one period) (a); Cartesian linear [m/s] and angular $[\mathrm{rad} / \mathrm{s}]$ velocity error (mean over one period) (b)

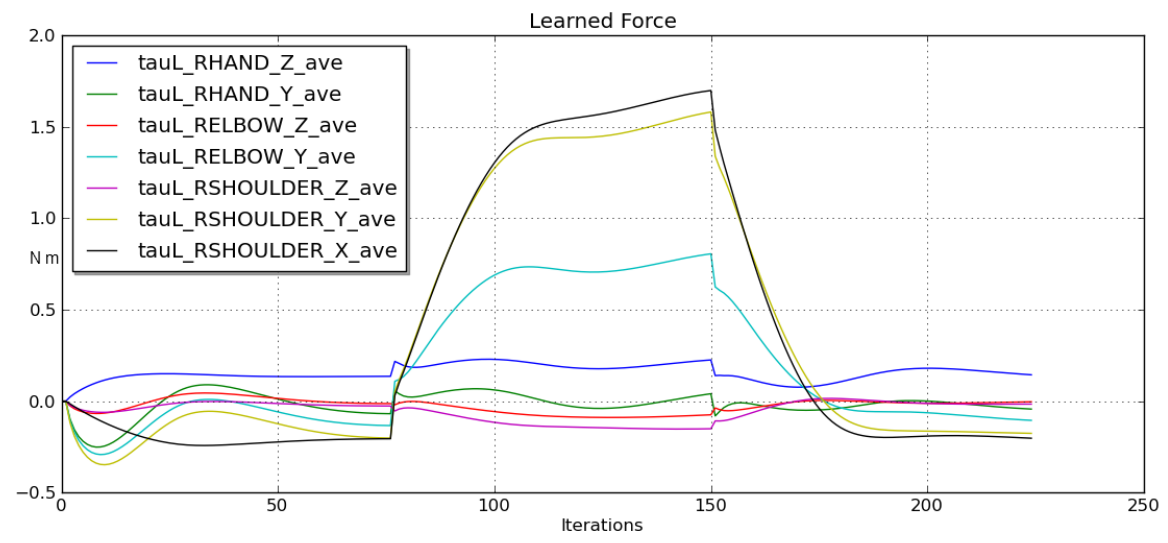

Fig. 7: Learned force (mean over one period) 


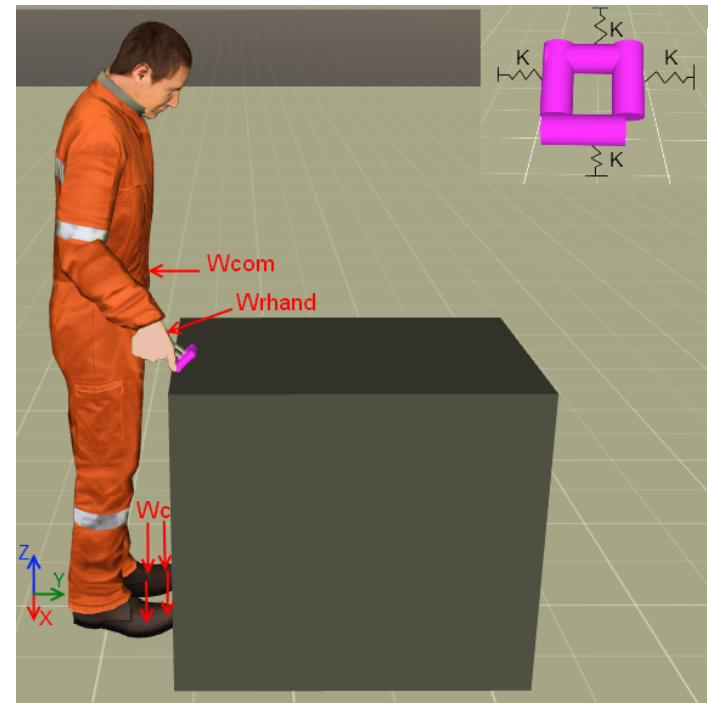

(a)

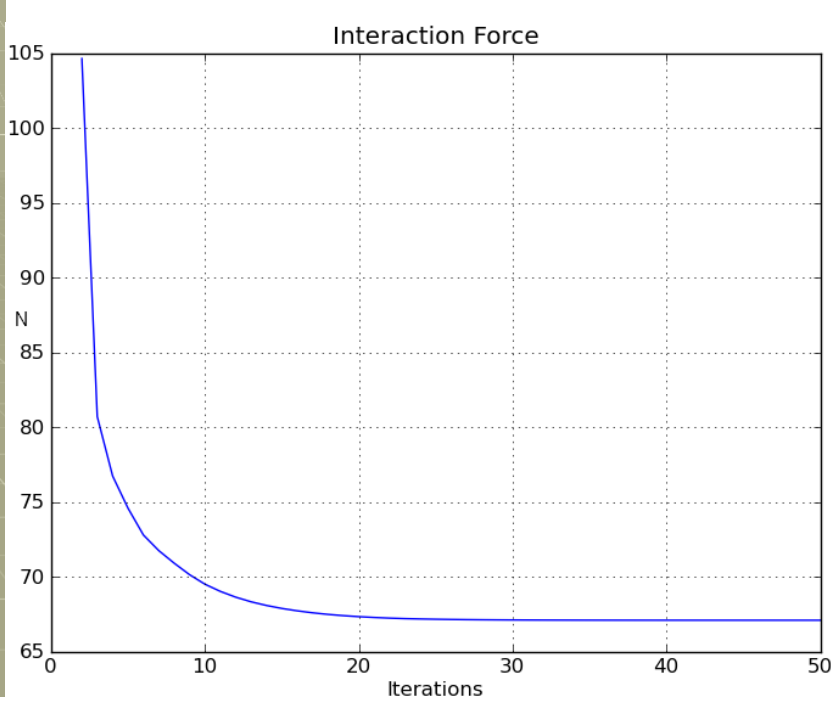

(b)

Fig. 8: DMU scenario and virtual object to model insertion with the stiffness $K_{o b j}$ of the object equal to $1000 \mathrm{~N} / \mathrm{m}$ in the four direction (a); Average interaction force during insertion (b);

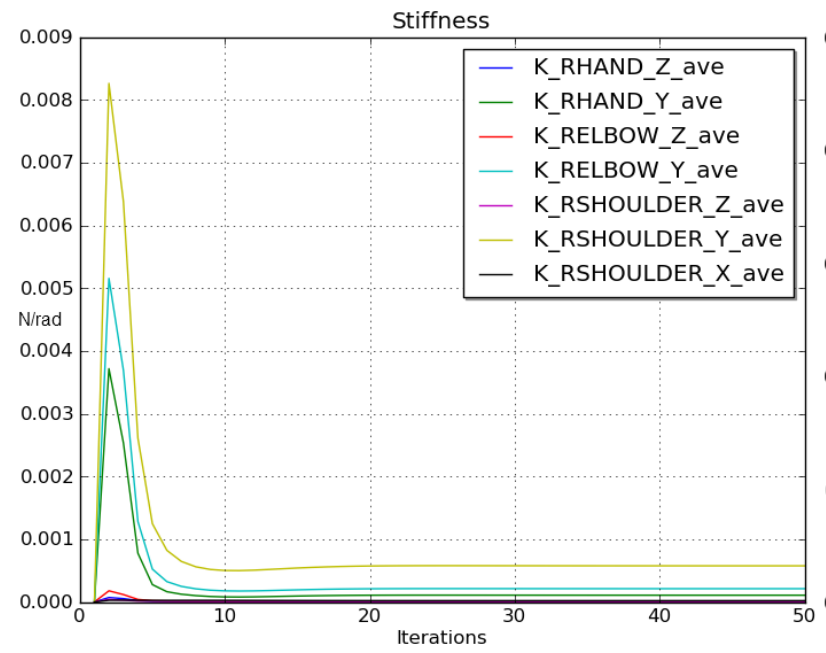

(a)

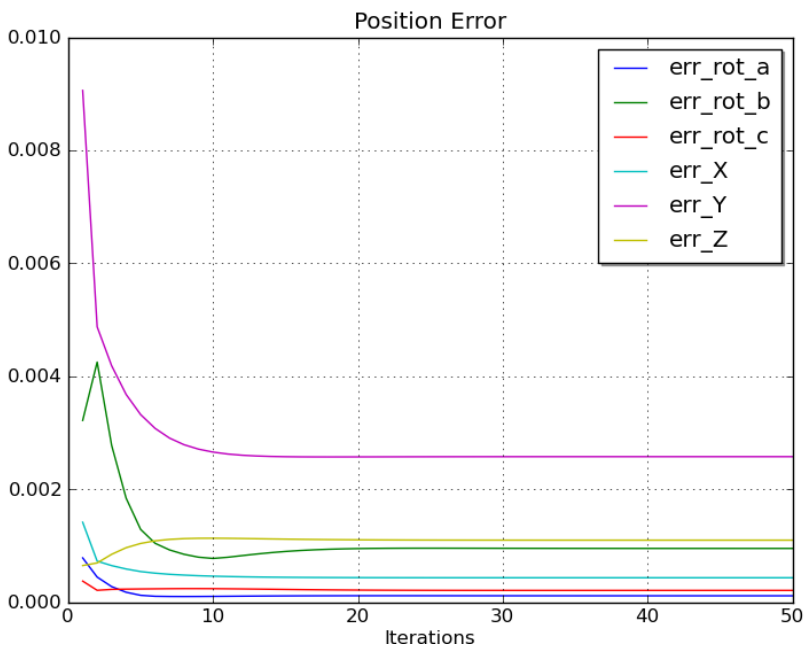

(b)

Fig. 9: Learned joint stiffness during insertion (mean over one period) (a); Cartesian position [m] and orientation [rad] error during insertion (mean over one period) (b)

10. Burdet, E., Osu, R., Franklin, D., Milner, T., Kawato, M.: The central nervous system stabilizes unstable dynamics by learning optimal impedance. Nature 414, 446-449 (2001)

11. Burdet, E., Tee, K.P., Mareels, I., Milner, T.E., Chew, C., Franklin, D.W., Osu, R., Kawato, M.: Stability and motor adaptation in human arm movements. Biological Cybernetics 94, 20-32 (1998)

12. Castillo, E.: Run-to-run process control: literature review and extensions. J. Qual. Technol. 29, 184-196 (1997)

13. Chaffin, D.: Human motion simulation for vehicle and workplace design. Human Factors and Ergonomics in Manufacturing 17, 475-484 (2007)
14. Cheah, C., Wang, D.: Learning impedance control for robotic manipulators. IEEE Transactions on Robotics and Automation 14, 452-465 (1998)

15. Colette, C., Micaelli, A., Andriot, C., Lemerle, P.: Robust balance optimization control of humanoid robots with multiple non coplanar grasps and frictional contacts. In: Proceedings of the IEEE International Conference on Robotics and Automation, pp. 3187-3193. Pasadena, USA (2008)

16. Commuri, S., Lewis, F.: Adaptive-fuzzy logic control of robot manipulators. In: IEEE International Conference on Robotics and Automation, vol. 3, pp. 2604-2609. Minneapolis, MN (1996) 
17. De Magistris, G.: Dynamic digital human model control design for the assessment of the workstation ergonomics. PhD Thesis - Pierre and Marie Curie University (2013)

18. De Magistris, G., Micaelli, A., Andriot, C., Savin, J., Marsot, J.: Dynamic virtual manikin control design for the assessment of the workstation ergonomy. In: First International Symposium on Digital Human Modeling. Lyon (2011)

19. De Magistris, G., Micaelli, A., Evrard, P., Andriot, C., Savin, J., Gaudez, C., Marsot, J.: Dynamic control of DHM for ergonomic assessments. International Journal of Industrial Ergonomics 43, 170-180 (2013)

20. De Magistris, G., Micaelli, A., Savin, J., Gaudez, C., Marsot, J.: Dynamic digital human model for ergonomic assessment based on human-like behaviour and requiring a reduced set of data for a simulation. In: Second International Digital Human Model Symposium 2013. Ann Arbor, USA (2013)

21. Fitts, P.: The information capacity of the human motor system in controlling the amplitude of movement. Journal of Experimental Psychology 47, 381-391 (1954)

22. Flash, T., Hogan, N.: The coordination of arm movements: an experimentally confirmed mathematical model. Journal of Neuroscience 7, 1688-1703 (1985)

23. Franklin, D., Burdet, E., Osu, R., Tee, K., Chew, C., Milner, T., Kawato, M.: CNS learns stable, accurate, and efficient movements using a simple algorithm. J Neuroscience 28, 11165-11173 (2008)

24. Ganesh, G., Albu-Schaeffer, A., Haruno, M., Kawato, M., Burdet, E.: Biomimetic motor behavior or simultaneous adaptation of force, impedance and trajectory in interaction tasks. In: IEEE International Conference on Robotics and Automation. Anchorage, Alaska, USA (2010)

25. Gomi, H., Osu, R.: Task-dependent viscoelasticity of human multijoint arm and its spatial characteristics for interaction with environments. Journal of Neuroscience 18, 8965-8978 (1998)

26. Gribble, P., Ostry, D.: Compensation for interaction torques during single and multijoint limb movement. Journal of Neurophysiology 82, 2310-2326 (1999)

27. Grossman, T., Balakrishnan, R.: Pointing at trivariate targets in 3d environments. In: Proceedings of the SIGCHI Conference on Human Factors in Computing Systems, pp. 447-454. New York (2004)

28. Haddadin, S., Albu-Schffer, A., Hirzinger, G.: Requirements for safe robots: Measurements, analysis \& new insights. International Journal on Robotics Research 28, 1507-1527 (2009)

29. Hanavan, E.: A mathematical model of the human body. Wright-Patterson Air Force Base Report No. AMRLTR102, 64-102 (1964)

30. Hogan, N.: Impedance control: an approach to manipulation-part i: Theory; part ii: Implementation; part iii: Applications. Transaction ASME J. Dynamic Systems, Measurement and Control 107, 11-24 (1985)

31. Hogan, N.: Mechanical impedance of single- and multiarticular systems. j. m. winters \& s.l. woo. springerverlag. Multiple muscle systems: Biomechanics and movement organization (1990)

32. Hovland, G., Sikka, P., McCarragher, B.: Skill acquisition from human demonstration using a hidden markov model. In: IEEE International Conference on Robotics and $\mathrm{Au}-$ tomation, vol. 3, pp. 2706-2711. Minneapolis, MN (1996)

33. Hsu, L.: Self-oscillating adaptive systems (soas) without limit-cycles. In: Proceedings of the American Control Conference, vol. 13. Albuquerque, New Mexico (1997)
34. Hyman, R.: Stimulus information as a determinant of reaction time. Journal of Experimental Psychology 45, 188196 (1953)

35. Ioannou, P., Kokotović, P.: Adaptive systems with reduced models. Lecture Notes in Control and Information Sciences 47 (1983)

36. Jagannathan, S.: Neural Network Control of Nonlinear Discrete-Time Systems. CRC Press Taylor \& Francis Group, FL (2006)

37. Kawato, M., Gomi, H.: A computational model of four regions of the cerebellum based on feedback error learning. Biological Cybernetics 69, 95-103 (1992)

38. Khatib, O., Sentis, L., Park, J., Warren, J.: Whole-body dynamic behavior and control of human-like robots. International Journal of Humanoid Robotics 01, 29-43 (2004)

39. Kirsch, R., Boskov, D., Rymer, W., Center, R., Center, M., Cleveland, O.: Muscle stiffness during transient and continuous movements of catmuscle: perturbation characteristics and physiological relevance. IEEE Transactions on Biomedical Engineering 41, 758-770 (1994)

40. Lackner, J., Dizio, P.: Rapid adaptation to coriolis force perturbations of arm trajectory. Journal of Neurophysiology 72, 299-313 (1994)

41. Li, C., Zhang, D., Zhuang, X.: A survey of repetitive control. In: Proceedings of 2004 IEEE/RSJ International Conference on Intelligent Robots and Systems, pp. 1160-1166. Sendai, Japan (2004)

42. Liu, M., Micaelli, A., Evrard, P., Escande, A., Andriot, C.: Interactive dynamics and balance of a virtual character during manipulation tasks. In: IEEE International Conference on Robotics and Automation, pp. 1676-1682. Shanghai, China (2011)

43. Lämkull, D., Hanson, L., Ortengren, R.: A comparative study of digital human modelling simulation results and their outcomes in reality: A case study within manual assembly of automobiles. International Journal of Industrial Ergonomics 39, 428-441 (2009)

44. McIntyre, J., Mussa-Ivaldi, F., Bizzi, E.: The control of stable arm postures in the multi-joint arm. Exp Brain Res, pp. 248-264 (1996)

45. Merlhiot, X.: A robust, efficient and time-stepping compatible collision detection method for non-smooth contact between rigid bodies of arbitrary shape. In: Proceedings of the Multibody Dynamics ECCOMAS Thematic Conference (2007)

46. Merlhiot, X.: Extension of a time-stepping compatible contact determination method between rigid bodies to deformable models. In: Proceedings of the Multibody Dynamics ECCOMAS Thematic Conference (2009)

47. Miller, A., Knoop, S., Christensen, H., Allen, P.: Automatic grasp planning using shape primitives. In: IEEE International Conference on Robotics and Automation, vol. 2, pp. 1824-1829 (2003)

48. Milner, T., Cloutier, C.: Compensation for mechanically unstable loading in voluntary wrist movement. Experimental Brain Research 94, 522-532 (1993)

49. Miyata, N., Kouki, M., Mochimaru, M., Kawachi, K., Kurihara, T.: Hand link modelling and motion generation from motion capture data based on 3d joint kinematics. In: Proceedings SAE International Iowa (2005)

50. Morasso, P.: Spatial control of arm movements. Experimental Brain Research 42, 223-227 (1981)

51. Morasso, P., Mussa-Ivaldi, F.: Trajectory formation and handwriting: a computational model. Biological Cybernetics 45, 131-142 (1982)

52. Morasso, P., Sanguineti, V.: Ankle stiffness alone cannot stabilize upright standing. Journal of Neurophysiology $\mathbf{8 8}$, 2157-2162 (2002) 
53. Occhipinti, E.: Ocra, a concise index for the assessment of exposure to repetitive movements of the upper limbs. Ergonomics 41, 1290-1311 (1998)

54. Perreault, E., Kirsch, R., Crago, P.: Multijoint dynamics and postural stability of the human arm. Experimental Brain Research 157, 507-517 (2004)

55. Porter, J.M., Case, K., Marshall, R., Gyi, D., Sims, R.: Beyond Jack and Jill: designing for individuals using HADRIAN. International Journal of Industrial Ergonomics 33, 249-264 (2004)

56. Prakash, N.: Differential Geometry, an Integrated Approach. TATA McGraw-Hill Publishing Company Limited (1981)

57. Pratt, J., Torres, A., Dilworth, P., Pratt, G.: Virtual actuator control. In: IEEE International Conference on Intelligent Robots and Systems, pp. 1219-1226 (1996)

58. Salini, J.: Dynamic control for the task/posture coordination of humanoids: toward synthesis of complex activities. Ph.D. thesis, University of Pierre and Marie Curie (2012)

59. Schaub, K., Landau, K., Menges, R., Grossmann, K.: A computer-aided tool for ergonomic workplace design and preventive health care. Human Factors and Ergonomics in Manufacturing 7, 269-304 (1997)

60. Sciavicco, L., Siciliano, B.: Modelling and Control of Robot Manipulators. Springer, London (2000)

61. Shadmehr, R., Mussa-Ivaldi, F.: Adaptive representation of dynamics during learning of a motor task. Journal of Neuroscience 14, 3208-3224 (1997)

62. Si, J., Zhang, N., Tang, R.: Modified fuzzy associative memory scheme using genetic algorithm. In: Proceedings of the 1999 Congress on Evolutionary Computation (CEC), vol. 3, pp. 2002-2006 (1999)

63. Slotine, J., Li, W.: Applied Nonlinear Control. PrenticeHall, Englewood Cliff, NJ (1991)

64. Smith, A.: Does the cerebellum learn strategies for the optimal time-varying control of joint stiffness? Behavioral and Brain Sciences 19, 399-410 (1996)

65. Tee, K., Franklin, D., Kawato, M., Milner, T., Burdet, E.: Concurrent adaptation of force and impedance in the redundant muscle system. Biological Cybernetics 102, 31$44(2010)$

66. Todorov, E., Jordan, M.: Smoothness maximization along a predefined path accurately predicts the speed profiles of complex arm movements. Journal of Neurophysiology 80, 697-714 (1998)

67. Uno, Y., Kawato, M., Suzuki, R.: Formation and control of optimal trajectory in human multijoint arm movement: Minimum torque-change model. Biological Cybernetics 61, 89-101 (1989)

68. Vignes, R.: Modeling Muscle Fatigue in Digital Humans. Center for Computer-Aided Design, The University of IOWA. Tech. rep. (2004)

69. VSR Research Group: Technical report for project virtual soldier research. Center for Computer-Aided Design, The University of IOWA. Tech. rep. (2004)

70. Wolpert, D., Miall, C., Kawato, M.: Internal models in the cerebellum. Trends Cognitive Sciences 2, 338-347 (1998)

71. Won, J., Hogan, N.: Stability properties of human reaching movements. Experimental Brain Research 107, 125-136 (1995)

72. Yang, C., Ganesh, G., Haddadin, S., Parusel, S., AlbuSchaeffer, A., Burdet, E.: Human like adaptation of force and impedance in stable and unstable interactions. Transactions on Robotics 27, 918-930 (2011) 


\section{A Relation between cartesian space and joint space}

Using Eqs. 1, the interaction dynamics is:

$M \dot{T}+N T+G=L \tau+J_{c}^{T} W_{c}+J_{e n d}^{T} W_{e n d}^{i}$

Given an interaction wrench $W_{e n d}^{i}$

In this paper, we treat the DHM control where the floating base is the foot. We consider cases with the foot fixed to the ground. In this way, we obtain a completely actuated DHM with fixed-base robots characteristics. The dynamic model of DHM is:

$M_{q} \ddot{q}+N_{q} \dot{q}+G_{q}=\tau+J_{c, q}^{T} W_{c}+J_{e n d, q}^{T} W_{e n d}^{i}$

with $M_{q}=L^{T} M L, N_{q}=L^{T} N L, G_{q}=L^{T} G, J_{c, q}^{T}=L^{T} J_{c}^{T}$ and $J_{e n d, q}^{T}=L^{T} J_{e n d}^{T}$. When the only contact with the ground is the foot and it is the root, we obtain $J_{c} L=0$.

Since $\rho=S q$ and $S$ is a matrix to select a part of the actuated degrees of freedom $(S=[I 0])$ to obtain a dyamic model independent of non-sliding contact forces at known fixed locations in Eq. 1 such as the contacts between the feet and the ground, we can write the system as:

$M_{\rho} \ddot{\rho}+N_{\rho} \dot{\rho}+G_{\rho}=\tau_{\rho}+J_{e n d, \rho}^{T} W_{e n d}^{i}$

with $M_{\rho}=S M_{q} S^{T}, N_{\rho}=S N_{q} S^{T}, G_{\rho}=S G_{q}$ and $J_{e n d, \rho}^{T}=S J_{e n d, q}^{T}$

From Eq. 35 and since $\delta W_{\text {end }}^{i}=K_{\text {end }} \operatorname{vec}\left(H_{\text {end }}^{-1} \delta H_{\text {end }}\right)=K_{\text {end }} J_{\text {end,q }} \delta q=K_{\text {end }} J_{\text {end,q } q} \delta\left(S^{t} \rho\right)=K_{\text {end }} J_{\text {end, } \rho} \delta \rho$, we obtain:

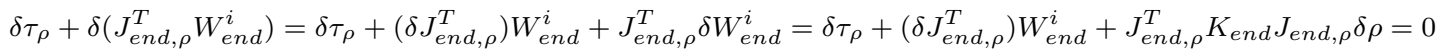

Since $\delta \tau_{\rho}=-K_{\rho} \delta \rho$ and Eq. 36, we obtain:

$K_{\rho}=-\frac{\delta \tau_{\rho}}{\delta \rho}=J_{e n d, \rho}^{T} K_{e n d} J_{e n d, \rho}+\frac{\partial J_{e n d, \rho}^{T}}{\partial \rho} W_{e n d}^{i}$

Finally, the cartesian impedance is:

$K_{\text {end }}=J_{\text {end, } \rho}^{\dagger T}\left(K_{\rho}-\frac{\partial J_{e n d, \rho}^{T}}{\partial \rho} W_{e n d}^{i}\right) J_{e n d, \rho}^{\dagger}$

with $J_{e n d, \rho}^{\dagger}$ the dynamic pseudo-inverse [38] defined as:

$J_{\text {end, } \rho}^{\dagger}=M_{\rho}^{-1} J_{e n d, \rho}^{T}\left(J_{\text {end, }, \rho} M_{\rho}^{-1} J_{e n d, \rho}^{T}\right)^{-1}$

It can be similarly obtained $B_{\text {end }}=J_{\text {end, } \rho}^{\dagger T} B_{\rho} J_{\text {end, } \rho}^{\dagger}$.

\section{B Convergence Analysis}

B.1 Motion error cost function

The first derivative of $M_{E}$ (Eq. 5) can be calculated as follows:

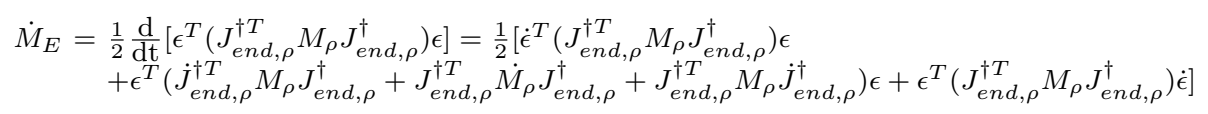

with

$\epsilon=V-V^{*}, \quad \dot{\epsilon}=A-A^{*}$

and $V^{*}=V^{d}-b \delta\left(H^{d}, H^{r}\right) . V^{d}$ is the velocity obtained by minimum jerk planner. $A^{*}$ is the derivative of $V^{*}$.

Matrix $M_{\rho}$ is symmetric, we therefore obtain:

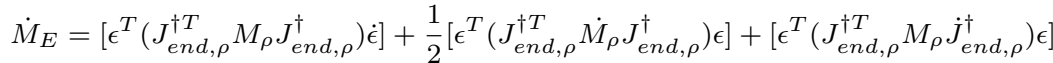

The relationship between $\rho$ velocity and Cartesian space velocity can be expressed as:

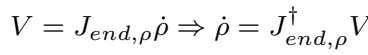

Differentiating Eq. 43, the cartesian acceleration term can be found as:

$A=J_{\text {end, } \rho} \ddot{\rho}+\dot{J}_{\text {end }, \rho} \dot{\rho}$ 
then the equation of robot motion in joint space can also be represented in Cartesian space coordinates by the relationship:

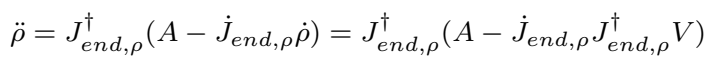

Substituting Eqs. 45 and 43 into Eq. 35 yields:

$M_{\rho} J_{e n d, \rho}^{\dagger}\left[A-\dot{J}_{\text {end, } \rho} J_{\text {end, },}^{\dagger} V\right]+N_{\rho} J_{\text {end, },}^{\dagger} V+G_{\rho}=\tau_{\rho}+J_{e n d, \rho}^{T} W_{e n d}^{i}$

Multiplying both side by $J_{e n d, \rho}^{\dagger T}$, we obtain:

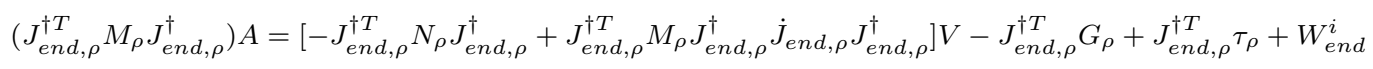

Using Eq. 10, we otbain:

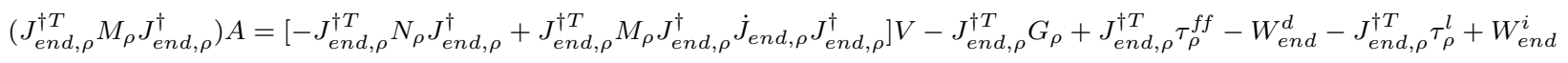

where $\tau_{\rho}^{f f}$ is the torque to compensate for DHM dynamics. By definition, it can be written as:

$\tau_{\rho}^{f f} \equiv M_{\rho} \ddot{\rho}^{*}+N_{\rho} \dot{\rho}^{*}+G_{\rho} \equiv M_{\rho} J_{e n d, \rho}^{\dagger} A^{*}+\left[N_{\rho} J_{e n d, \rho}^{\dagger}-M_{\rho} J_{e n d, \rho}^{\dagger} \dot{J}_{e n d, \rho} J_{e n d, \rho}^{\dagger}\right] V^{*}+G_{\rho}$

Using Eq. 41 and substituting Eq. 49 into Eq. 48 yields:

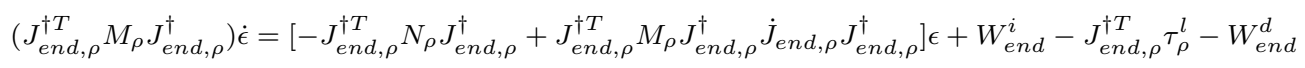

Substituting Eq. 50 into Eq. 42 yields:

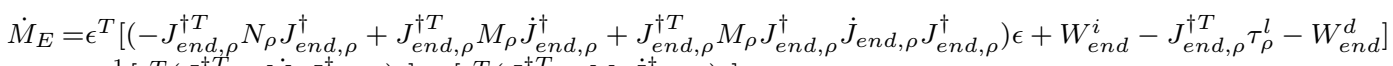

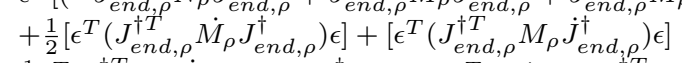

$$
\begin{aligned}
& =\frac{1}{2} \epsilon^{T}\left[J_{e n d, \rho}^{\dagger T}\left(\dot{M}_{\rho}-2 N_{\rho}\right) J_{e n d, \rho}^{\dagger}\right] \epsilon+\epsilon^{T}\left[W_{e n d}^{i}-J_{e n d, \rho}^{\dagger T} \tau_{\rho}^{l}-W_{e n d}^{d}\right]+\epsilon^{T}\left[J_{e n d, \rho}^{\dagger T} M_{\rho} \dot{J}_{e n d, \rho}^{\dagger}+J_{e n d, \rho}^{\dagger T} M_{\rho} J_{e n d, \rho}^{\dagger} \dot{J}_{\text {end, }, \rho} J_{e n d, \rho}^{\dagger}\right] \epsilon
\end{aligned}
$$

Matrix $\dot{M}_{\rho}-2 N$ is skew-symmetry [60] and for this reason, we have:

$\epsilon^{T}\left(J_{\text {end, } \rho}^{\dagger T}\left(\dot{M}_{\rho}-2 N_{\rho}\right) J_{\text {end, } \rho}^{\dagger}\right) \epsilon=0$

Let us now analyze the third term of Eq. 51. Using Eq. 39, since $J_{e n d, \rho} J_{e n d, \rho}^{\dagger}=I$ and $\dot{J}_{\text {end, }, \rho}^{\dagger} J_{e n d, \rho}^{\dagger}+J_{\text {end, }, \rho} \dot{J}_{\text {end, }, \rho}^{\dagger}=0$, we obtain:

$J_{e n d, \rho}^{\dagger T} M_{\rho} \dot{J}_{e n d, \rho}^{\dagger}=\left(J_{\text {end, },} M_{\rho}^{-1} J_{e n d, \rho}^{T}\right)^{-1} J_{\text {end, },} M_{\rho}^{-1} M_{\rho} \dot{J}_{\text {end, } \rho}^{\dagger}=\left(J_{\text {end, }, \rho} M_{\rho}^{-1} J_{\text {end, },}^{T}\right)^{-1} J_{\text {end, },} \dot{J}_{\text {end }, \rho}^{\dagger}$

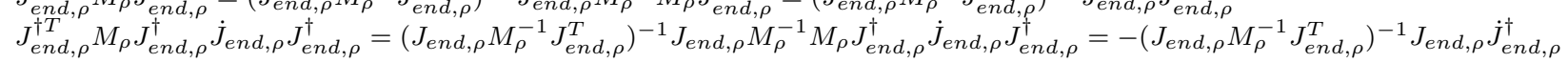

Substituting Eq. 52 and Eq. 53 into Eq. 51, we obtain:

$\dot{M}_{E}=\epsilon^{T}\left[W_{e n d}^{i}-J_{e n d, \rho}^{\dagger T} \tau_{\rho}^{l}-W_{e n d}^{d}\right]$

Using Eqs. 54, 11 and 38, we have:

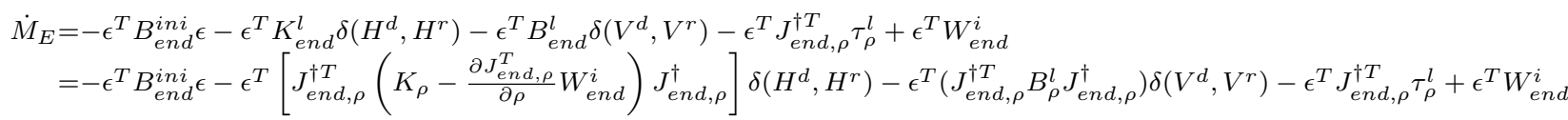

We can derive $\delta M_{E}(t)=M_{E}(t)-M_{E}(t-D)$ from Eqs. 55 and 8 as:

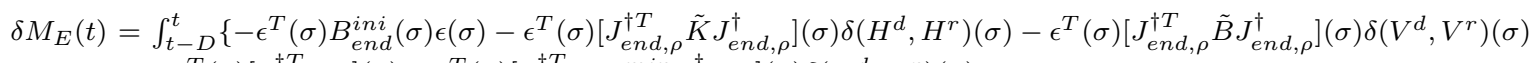

$$
\begin{aligned}
& -\epsilon^{T}(\sigma)\left[J_{\text {end }}^{\dagger T} \tilde{\tau}\right](\sigma)-\epsilon^{T}(\sigma)\left[J_{\text {end, } \rho}^{\dagger T} K_{\rho}^{m i n} J_{\text {end, },}^{\dagger}\right](\sigma) \delta\left(H^{d}, H^{r}\right)(\sigma)
\end{aligned}
$$

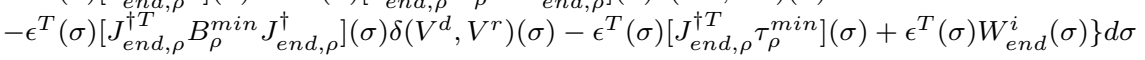

Any smooth interaction force can be approximated by the linear terms of its Taylor expansion along the reference trajectory as follows:

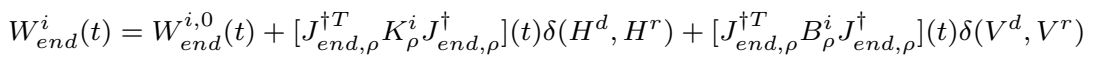

where $W_{\text {end }}^{i, 0}$ is the zero order term compensated by $J_{e n d, \rho}^{\dagger T} \tau^{m i n} ;\left[J_{e n d}^{\dagger T} K_{\rho}^{i} J_{e n d}^{\dagger}\right]$ and $\left[J_{e n d}^{\dagger T} B_{\rho}^{i} J_{e n d}^{\dagger}\right]$ are the first order coefficients. From Eqs. 57 and 41, we can obtain the values for $K_{\rho}^{\min }(t), B_{\rho}^{\min }(t)$ and $\tau_{\rho}^{\min }(t)$ to guarantee stability (Eq. 58). Different $W_{e n d}^{i}$ will yield different values of $K_{\rho}^{\min }(t), B_{\rho}^{\min }(t)$ and $\tau_{\rho}^{\min }(t)$ and when $W_{e n d}^{i}$ is zero or is assisting the tracking task $\|\epsilon(t)\| \rightarrow 0, K_{\rho}^{m i n}(t)$, $B_{\rho}^{\min }(t)$ and $\tau_{\rho}^{\min }(t)$ will be 0 .

$K_{\rho}^{\min }(t), D_{\rho}^{\min }(t)$ and $\tau_{\rho}^{\text {min }}(t)$ represent the minimal required effort of stiffness, damping and feedforward force required to guarantee

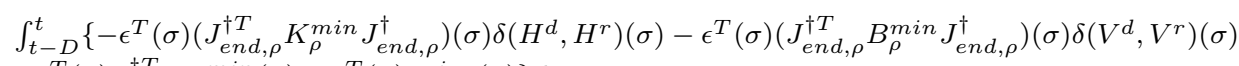

$\left.-\epsilon^{T}(\sigma) J_{\text {end, } \rho}^{\dagger T} \tau_{\rho}^{m i n}(\sigma)+\epsilon^{T}(\sigma) W_{\text {end }}^{i}(\sigma)\right\} d \sigma \leq 0$

so that from Eq. 55 we have $\int_{t-D}^{t} \dot{M}_{E}(\sigma) d \sigma \leq 0$.

From Eqs. 56 and 58, we can write:

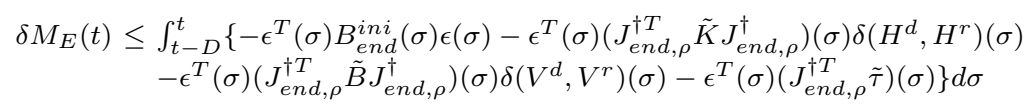


B.2 Metabolic cost function

The metabolic cost function is:

$M_{C}(t)=\frac{1}{2} \int_{t-D}^{t} \tilde{\Phi}^{T}(\sigma) Q^{-1} \tilde{\Phi}(\sigma) d \sigma$

According to the definition of $\Phi(t)$ and $Q$, the following properties of $\operatorname{vec}(\cdot), \otimes$ and $\operatorname{tr}(\cdot)$ operators:

$\operatorname{vec}(\Omega Y U)=\left(U^{T} \otimes \Omega\right) \operatorname{vec}(Y), \operatorname{tr}(\Omega Y)=\operatorname{vec}\left(\Omega^{T}\right)^{T} \operatorname{vec}(Y), \operatorname{tr}(\Omega Y)=\operatorname{tr}(Y \Omega)$

and using the symmetry of $Q_{K}^{-1}$, we obtain:

$\operatorname{vec}\left(\tilde{K}^{T}\right)^{T}\left(I \otimes Q_{K}\right)^{-1} \operatorname{vec}\left(\tilde{K}^{T}\right)=\operatorname{vec}\left(\tilde{K}^{T}\right)^{T}\left(\left(Q_{K}^{-1}\right)^{T} \otimes I\right) \operatorname{vec}\left(\tilde{K}^{T}\right)=\operatorname{vec}\left(\tilde{K}^{T}\right)^{T} \operatorname{vec}\left(\tilde{K}^{T} Q_{K}^{-1}\right)=\operatorname{tr}\left\{\tilde{K} \tilde{K}^{T} Q_{K}^{-1}\right\}=\operatorname{tr}\left\{\tilde{K}^{T} Q_{K}^{-1} \tilde{K}\right\}$

In the same way, can be found the terms corresponding to $\tilde{B}$ and $\tilde{\tau}$.

For these reasons, we can define $\delta M_{C}(t)=M_{C}(t)-M_{C}(t-D)$ as:

$\delta M_{C}(t)=\frac{1}{2} \int_{t-D}^{t}\left\{\operatorname{tr}\left\{\left[\tilde{K}^{T}(\sigma) Q_{K}^{-1} \tilde{K}(\sigma)\right]-\left[\tilde{K}^{T}(\sigma-D) Q_{K}^{-1} \tilde{K}(\sigma-D)\right]\right\}+\operatorname{tr}\left\{\left[\tilde{B}^{T}(\sigma) Q_{B}^{-1} \tilde{B}(\sigma)\right]-\left[\tilde{B}^{T}(\sigma-D) Q_{B}^{-1} \tilde{B}(\sigma-D)\right]\right\}\right.$

From Eqs. 13, 14 and 16, we obtain:

$\delta K=Q_{K}\left\{J_{e n d, \rho}^{\dagger}\left[\epsilon(t) \delta\left(H^{d}, H^{r}\right)^{T}\right] J_{e n d, \rho}^{\dagger T}-\gamma(t) K_{\rho}^{l}(t)\right\}$

$\delta B=Q_{B}\left\{J_{\text {end, },}^{\dagger}\left[\epsilon(t) \delta\left(V^{d}, V^{r}\right)^{T}\right] J_{\text {end, }, \rho}^{\dagger T}-\gamma(t) B_{\rho}^{l}(t)\right\}$

$\delta \tau=Q_{\tau}\left\{J_{\text {end, } \rho}^{\dagger} \epsilon(t)-\gamma(t) \tau_{\rho}^{l}(t)\right\}$

Using the symmetry of $Q_{K}^{-1}, \tilde{K}(\sigma)-\tilde{K}(\sigma-D)=\delta K(\sigma)$ and Eq. 64, the first term in the integrand of Eq. 63 can be written as:

$\operatorname{tr}\left\{\left[\tilde{K}^{T}(\sigma) Q_{K}^{-1} \tilde{K}(\sigma)\right]-\left[\tilde{K}^{T}(\sigma-D) Q_{K}^{-1} \tilde{K}(\sigma-D)\right]\right\}=\operatorname{tr}\left\{\left[\tilde{K}^{T}(\sigma)-\tilde{K}^{T}(\sigma-D)\right]^{T} Q_{K}^{-1}\left[2 \tilde{K}^{T}(\sigma)-\tilde{K}^{T}(\sigma)+\tilde{K}^{T}(\sigma-D)\right]\right\}$

$=\operatorname{tr}\left\{\delta K^{T}(\sigma) Q_{K}^{-1}[2 \tilde{K}(\sigma)-\delta K(\sigma)]\right\}=-\operatorname{tr}\left\{\delta K^{T}(\sigma) Q_{K}^{-1} \delta K(\sigma)\right\}+2 \operatorname{tr}\left\{\delta K(\sigma) Q_{K}^{-1} \tilde{K}(\sigma)\right\}$

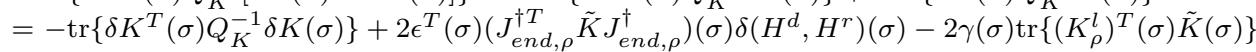

In the same way, can be found the second terms in the integrand of Eq. 63 as:

$\operatorname{tr}\left\{\tilde{B}^{T}(\sigma) Q_{B}^{-1} \tilde{B}(\sigma)-\tilde{B}^{T}(\sigma-D) Q_{B}^{-1} \tilde{B}(\sigma-D)\right\}$

$=-\operatorname{tr}\left\{\delta B^{T}(\sigma) Q_{B}^{-1} \delta B(\sigma)\right\}+2 \epsilon^{T}(\sigma)\left(J_{\text {end, }, ~}^{\dagger T} \tilde{B} J_{\text {end, }, \rho}^{\dagger}\right)(\sigma) \delta\left(H^{d}, H^{r}\right)(\sigma)-2 \gamma(\sigma) \operatorname{tr}\left\{\left(B_{\rho}^{l}\right)^{T}(\sigma) \tilde{B}(\sigma)\right\}$

and third terms in the integrand of Eq. 63 as:

$\left[\tilde{\tau}^{T}(\sigma) Q_{\tau}^{-1} \tilde{\tau}(\sigma)\right]-\left[\tilde{\tau}^{T}(\sigma-D) Q_{\tau}^{-1} \tilde{\tau}(\sigma-D)\right]=-\left[\delta \tau^{T}(\sigma) Q_{\tau}^{-1} \delta \tau(\sigma)\right]+2 \epsilon^{T}(\sigma)\left(J_{\text {end, }, ~}^{\dagger T} \tilde{\tau}\right)(\sigma)-2 \gamma(\sigma)\left(\tau_{\rho}^{l}\right)^{T}(\sigma) \tilde{\tau}(\sigma)$

Replacing Eqs. 65, 66 and 67 into 63, we obtain:

$\delta M_{C}(t)=-\frac{1}{2} \int_{t-D}^{t}\left[\delta \tilde{\Phi}^{T}(\sigma) Q^{-1} \delta \tilde{\Phi}(\sigma)\right] d \sigma-\int_{t-D}^{t}\left[\gamma(\sigma) \tilde{\Phi}^{T}(\sigma) \Phi(\sigma)\right] d \sigma$

$$
+\int_{t-D}^{t}\left[\epsilon^{T}(\sigma)\left(J_{e n d, \rho}^{\dagger T} \tilde{K} J_{e n d, \rho}^{\dagger}\right)(\sigma) \delta\left(H^{d}, H^{r}\right)(\sigma)+\epsilon^{T}(\sigma)\left(J_{e n d, \rho}^{\dagger T} \tilde{B} J_{e n d, \rho}^{\dagger}\right)(\sigma) \delta\left(V^{d}, V^{r}\right)(\sigma)+\epsilon^{T}(\sigma)\left(J_{e n d, \rho}^{\dagger T} \tilde{\tau}\right)(\sigma)\right] d \sigma
$$

Combining Eqs. 59 and 68, we obtain the first difference of cost function:

$\delta C(t)=C(t)-C(t-D)=\delta M_{E}(t)+\delta M_{C}(t)$

$$
\leq-\frac{1}{2} \int_{t-D}^{t}\left[\delta \tilde{\Phi}^{T}(\sigma) Q^{-1} \delta \tilde{\Phi}(\sigma)\right] d \sigma-\int_{t-D}^{t}\left[\gamma(\sigma) \tilde{\Phi}^{T}(\sigma) \tilde{\Phi}+\gamma(\sigma) \tilde{\Phi}^{T}(\sigma) \Phi^{d}(\sigma)+\epsilon^{T}(\sigma) B_{\text {end }}^{\text {ini }}(\sigma) \epsilon(\sigma)\right] d \sigma
$$

To obtain $\delta C(t) \leq 0$, a sufficient condition is:

$\epsilon^{T} B_{\text {end }}^{i n i} \epsilon+\gamma \tilde{\Phi}^{T} \tilde{\Phi}+\gamma \tilde{\Phi}^{T} \Phi^{d} \geq \lambda_{B}\|\epsilon\|^{2}+\gamma\|\tilde{\Phi}\|^{2}-\gamma\|\tilde{\Phi}\|\left\|\Phi^{d}\right\| \geq 0$

where $\lambda_{B}$ as the infimum of the smallest eigenvalue of $B_{\text {end }}^{\text {ini }}$.

Replacing $\gamma(t)=\frac{p}{1+u|| \epsilon(t) \|^{2}}$ into Eq. 70, we obtain:

$\lambda_{B} u\|\epsilon\|^{4}+\lambda_{B}\|\epsilon\|^{2}+p\|\tilde{\Phi}\|^{2}-p\left\|\tilde{\Phi}|\||\left|\Phi^{d}\right| \mid \geq 0\right.$

To find the regions of points $\left(\|\epsilon\|^{2},\|\tilde{\Phi}\|\right)$ for each of which Eq. 71 holds, we need firstly to determine the set of points that satisfies:

$\lambda_{B} u\|\epsilon\|^{4}+\lambda_{B}\|\epsilon\|^{2}+p\|\tilde{\Phi}\|^{2}-p\left\|\tilde{\Phi}\left|\left\||| \Phi^{d}\right\|=0\right.\right.$

Eq. 72 is an ellipse passing trough the points $\left(\|\epsilon\|^{2}=0,\|\tilde{\Phi}\|=0\right)$ and $\left(\|\epsilon\|^{2}=0,\|\tilde{\Phi}\|=\| \Phi^{d}||\right)$.

To find the canonical equation of this ellipse, we need only to complete the squares and we obtain:

$\frac{\lambda_{B} u\left(\|\epsilon\|^{2}+\frac{1}{2 u}\right)^{2}+p\left(\|\tilde{\Phi}\|-\frac{\left\|\Phi^{d}\right\|}{2}\right)^{2}}{\frac{\lambda_{B}}{4 u}+\frac{p \| \Phi^{d}||^{2}}{4}}=1$ 
By Krasovskii-LaSalle principle, $\|\epsilon\|^{2}$ and $\|\tilde{\Phi}\|$ will converge to an invariant set $\Omega_{s} \subseteq \Omega$ on which $\delta C(t)=0$, where $\Omega$ is the bounding set defined as:

$\Omega \equiv\left\{\left(\|\epsilon\|^{2},\|\tilde{\Phi}\|\right), \frac{\lambda_{B} u\left(\|\epsilon\|^{2}+\frac{1}{2 u}\right)^{2}+p\left(\|\tilde{\Phi}\|-\left\|\Phi^{d}\right\| / 2\right)^{2}}{\frac{\lambda_{B}}{4 u}+\frac{p \| \Phi^{d}||^{2}}{4}} \leq 1\right\}$

If the parameter $\gamma$ is constant [24], the bounding set is:

$\left\{\left(\|\epsilon\|^{2},\|\tilde{\Phi}\|\right), \frac{4 \lambda_{B}\|\epsilon\|^{2}+4 \gamma\left(\|\tilde{\Phi}\|-\left\|\Phi^{d}\right\| / 2\right)^{2}}{\gamma\left\|\Phi^{d}\right\|^{2}} \leq 1\right\}$

$\gamma$ does not affect convergence, but the convergence speed and size of convergence set.

\section{Minjerk}

\section{C.1 Formal definition}

Using Eq. 20, we can write the inside term of the integral as:

$P=\left\|\frac{d^{3}}{d t^{3}} \mathbf{r}(s)\right\|^{2}=\left\|\frac{d^{2}}{d t^{2}} \mathbf{r}^{\prime}(s) \dot{s}\right\|^{2}=\left\|\frac{d}{d t}\left(\mathbf{r}^{\prime \prime}(s) \dot{s}^{2}+\mathbf{r}^{\prime}(s) \ddot{s}\right)\right\|^{2}=\left\|\mathbf{r}^{\prime \prime \prime}(s) \dot{s}^{3}+3 \mathbf{r}^{\prime \prime}(s) \dot{s} \ddot{s}+\mathbf{r}^{\prime}(s) \dddot{s}\right\|^{2}$

To explicit the invariance with respect to rotations and translations of the minimization problem in Eq. 76 , we can define uniquely $3 \mathrm{D}$ curve [56] by its curvature $R(s)$ and its torsion $\eta(s)$.

The path $\mathbf{r}$ satisfies Frenet's formulas:

$\mathbf{t}=R \mathbf{n} \mathbf{n}^{\prime}=\eta \mathbf{b}-R \mathbf{t} \mathbf{b}^{\prime}=-\eta \mathbf{n}$

From geometry, we know that:

$\mathbf{r}^{\prime}=\mathbf{t}^{\prime} \mathbf{r}^{\prime \prime}=R \mathbf{n} \mathbf{r}^{\prime \prime \prime}=R^{\prime} \mathbf{n}+R(\eta \mathbf{b}-R \mathbf{t})$

We replacing Eq. 78 in Eq. 76 and we obtain:

$P=\left\|\mathbf{n}\left(R^{\prime} \dot{s}^{3}+3 R \dot{s} \ddot{s}\right)+\mathbf{t}\left(\dddot{s}-R^{2} \dot{s}^{3}\right)+\mathbf{b}\left(\dot{s}^{3} R \eta\right)\right\|^{2}$

$\mathbf{n}, \mathbf{t}$ and $\mathbf{b}$ are orthogonal and thus we obtain:

$P=\left(R^{\prime} \dot{s}^{3}+3 R \dot{s} \ddot{s}\right)^{2}+\left(\dddot{s}-R^{2} \dot{s}^{3}\right)^{2}+\left(\dot{s}^{3} R \eta\right)^{2}$

\section{C.2 Relation to the $2 / 3$ power law}

We want to find the relation of Eq. 80 to $2 / 3$ power law.

To obtain this, we define a function:

$Z_{s}=\dot{s}^{3} R(s)$

$Z_{s}$ corresponds to the term multiplying the torsion $\eta$ in Eq. 80

We derive Eq. 81 respect to time and we obtain:

$R^{\prime}(s) \dot{s}^{4}+3 \dot{s}^{2} \ddot{s} R(s)=Z_{s}^{\prime} \dot{s}$

$R^{\prime}(s) \dot{s}^{3}+3 \dot{s} \ddot{s} R(s)=Z_{s}^{\prime}$

The term $R^{\prime}(s) \dot{s}^{3}+3 \dot{s} \ddot{s} R(s)$ is equal to the term multiplying $\mathbf{n}$ in Eq. 79 . We now substitute Eq. 82 in Eq. 79 :

$P=\left\|\mathbf{n}\left(Z_{s}^{\prime}\right)+\mathbf{t}\left(\dddot{s}-Z_{s} R\right)+\mathbf{b}\left(Z_{s} \eta\right)\right\|^{2}=Z_{s}^{\prime 2}+\left(\dddot{s}-Z_{s} R\right)^{2}+Z_{s}^{2} \eta^{2}$

From Eq. 81, we have:

$\dot{s}(t)=Z_{s}^{\frac{1}{3}} R^{-\frac{1}{3}}$

In the $2 / 3$ power law $Z_{s}^{\frac{1}{3}}=$ const and $Z_{s}^{\prime}=0$ and it is equivalent to setting the coefficient of $\mathbf{n}$ of the instantaneous jerk to zero, and the coefficient of $\mathbf{b}$ proportional to the coefficient of $\mathbf{t}$. To demonstrate this, we analyze the $2 \mathrm{D}$ power law:

$\left(x^{\prime 2}+y^{\prime 2}\right)^{1 / 2}=$ const $\left(\frac{\sqrt{\left.\left(x^{\prime} y^{\prime \prime}-y^{\prime} x^{\prime \prime}\right)^{2}\right)}}{\left(x^{\prime 2}+y^{\prime 2}\right)^{3 / 2}}\right) \Rightarrow x^{\prime} y^{\prime \prime}-y^{\prime} x^{\prime \prime}=$ const

Taking derivatives, we obtain:

$\frac{x^{\prime}}{y^{\prime}}=\frac{x^{\prime \prime \prime}}{y^{\prime \prime \prime}}, \quad \mathbf{r}^{\prime}=\mathbf{r}^{\prime \prime \prime}$

The jerk vector points is orthogonal to $\mathbf{n}$ and aligned with $\mathbf{t}$. Thus, the jerk along $\mathbf{n}$ is zero. 